Corrected: Publisher Correction

ARTICLE

https://doi.org/10.1038/s42003-019-0281-1

OPEN

\title{
SnRK2 protein kinases represent an ancient system in plants for adaptation to a terrestrial environment
}

\author{
Akihisa Shinozawa 1 , Ryoko Otake ${ }^{1}$, Daisuke Takezawa ${ }^{2}$, Taishi Umezawa ${ }^{3}$, Kenji Komatsu ${ }^{4}$, Keisuke Tanaka ${ }^{5}$, \\ Anna Amagai ${ }^{3}$, Shinnosuke Ishikawa ${ }^{3}$, Yurie Hara ${ }^{3}$, Yasuko Kamisugi ${ }^{6}$, Andrew C. Cuming (1) ${ }^{6}$, Koichi Hori (D) ${ }^{7}$, \\ Hiroyuki Ohta ${ }^{7}$, Fuminori Takahashi (1) ${ }^{8}$, Kazuo Shinozaki ${ }^{8}$, Takahisa Hayashi ${ }^{1}$, Teruaki Taji ${ }^{1}$ \& Yoichi Sakata (D) ${ }^{1}$
}

The SNF1-related protein kinase 2 (SnRK2) family includes key regulators of osmostress and abscisic acid ( $A B A$ ) responses in angiosperms and can be classified into three subclasses. Subclass III SnRK2s act in the ABA response while ABA-nonresponsive subclass I SnRK2s are regulated through osmostress. Here we report that an ancient subclass III SnRK2-based signalling module including ABA and an upstream Raf-like kinase (ARK) exclusively protects the moss Physcomitrella patens from drought. Subclass III SnRK2s from both Arabidopsis and from the semiterrestrial alga Klebsormidium nitens, which contains all the components of ABA signalling except ABA receptors, complement Physcomitrella snrk2- mutants, whereas Arabidopsis subclass I SnRK2 cannot. We propose that the earliest land plants developed the ABA/ARK/subclass III SnRK2 signalling module by recruiting ABA to regulate a pre-existing dehydration response and that subsequently a novel subclass I SnRK2 system evolved in vascular plants conferring osmostress protection independently from the ancient system.

\footnotetext{
${ }^{1}$ Department of Bioscience, Tokyo University of Agriculture, 1-1-1 Sakuragaoka, Setagayaku, Tokyo 156-8502, Japan. ${ }^{2}$ Graduate School of Science and Engineering, Saitama University, 255 Shimo-Okubo,Sakura-ku, Saitama 338-8570, Japan. ${ }^{3}$ Graduate School of Bio-Applications and Systems Engineering, Tokyo University of Agriculture and Technology, 2-24-16 Nakacho, Koganei, Tokyo 184-8588, Japan. ${ }^{4}$ Department of Bioproduction Technology, Junior College, Tokyo University of Agriculture, 1-1-1 Sakuragaoka, Setagayaku, Tokyo 156-8502, Japan. ${ }^{5}$ NODAI Genome Research Center (NGRC), Tokyo University of Agriculture, 1-1-1 Sakuragaoka, Setagayaku, Tokyo 156-8502, Japan. ${ }^{6}$ Centre for Plant Science, University of Leeds, Leeds LS2 9JT, UK. ${ }^{7}$ School of Life Science and Technology, Tokyo Institute of Technology, 4259 Nagatsuta-cho, Midori-ku, Yokohama, Kanagawa 226-8503, Japan. ${ }^{8}$ Gene Discovery Research Group, RIKEN Center for Sustainable Resource Science, 3-1-1, Koyadai, Tsukuba, Ibaraki 305-0074, Japan. These authors contributed equally: Akihisa Shinozawa, Ryoko Otake. Correspondence and requests for materials should be addressed to Y.S. (email: sakata@nodai.ac.jp)
} 
D ue to their sessile nature, land plants have developed various strategies to cope with dehydration under waterdeficit conditions. In addition to anatomical adaptations such as development of roots, vascular systems and cuticle-coated epidermal layers with stomata for efficient water usage, land plants also developed signal transduction systems for sensing and responding to water-deficient conditions. Osmostress-responsive accumulation of the phytohormone ABA triggers responses such as stomatal closure, and accumulation of late embryogenesis abundant (LEA) proteins and soluble sugars that protect cellular functions from dehydration ${ }^{1}$. ABA signalling acts through protein phosphorylation via SnRK2 kinases $^{2-5}$. These are classified in three subclasses (I-III $)^{6}$, and the subclass III SnRK2s are an essential component for ABA signal transduction regulated by soluble ABA receptors (PYR/PYL/RCAR) and Group A protein phosphatase 2Cs (PP2CAs) in angiosperms ${ }^{7,8}$. PP2CAs of Arabidopsis negatively regulate $\mathrm{ABA}$ signalling by inhibiting subclass III SnRK2 activity through direct interaction. The inhibition is cancelled when ABA binds to PYR/PYL/RCAR, which specifically sequester the PP2CAs, facilitating SnRK2 activation ${ }^{2,9,10}$. SnRK2s are also activated by osmostress. Disruption of all three subclasses in Arabidopsis results in a loss of osmostress responses ${ }^{11}$. However, it is yet to be elucidated how ABA-nonresponsive SnRK2 (subclass I) is regulated through osmostress.

Although SnRK2s are evolutionarily conserved among land plants $^{12}$, our knowledge of SnRK2 functions in non-angiosperms is limited. The genome of the model moss Physcomitrella patens encodes only four $S n R K 2$ genes (PpSnRK2A / 2B / 2C / 2D), all of which belong to subclass III ${ }^{13}$. PpSnRK2A/ Pp OPEN STOMATA1 (OST1) restores ABA responsiveness of stomatal closure in the Arabidopsis snrk2.6/ost1 mutant, and disruption of PpSnRK2A/ PpOST1 results in defective ABA-responsive closure of moss stomata, which occur only at the base of sporophytes, the nondominant generation in bryophytes ${ }^{14}$. However, it is not known whether the Ppsnrk2a/Ppost1 plant also has a defect in the ABA responsiveness of the moss protonemata, where $\mathrm{ABA}$ responses as well as tolerance to desiccation and osmostress have been well documented ${ }^{15-19}$.

Our previous studies have highlighted the differential regulation of ABA signalling between P. patens and Arabidopsis. We have shown that PP2CAs of $P$. patens play a major role in repressing ABA signalling downstream of the $40 \mathrm{kDa} A B A-$ activated kinases, suggesting a novel mechanism to regulate the kinase ${ }^{20}$. In addition, we identified a novel ABA-activated Raflike kinase (ARK), also known as ABA NON-RESPONSIVE ${ }^{21}$ or CONSTITUTIVE TRIPLE RESPONSE1-LIKE ${ }^{22}$, that acts upstream of the $40 \mathrm{kDa} \mathrm{ABA}$-activated kinases and is essential for ABA responses in $P$. patens ${ }^{21,23}$. In this study, to understand the function of SnRK2s in bryophytes, we establish Ppsnrk2 null mutants of $P$. patens using sequential gene targeting. We show that a high level of osmostress tolerance in $P$. patens is achieved exclusively through an ABA-SnRK2 signalling module. The highly conserved motif in the activation loop of land plant subclass III SnRK2s, which is the phosphorylation target of $\mathrm{ARK}^{23}$, plays a crucial role in SnRK2 activation. A cross-species complementation assay demonstrates that a $S n R K 2$ gene from the semiterrestrial alga Klebsormidium nitens, which contains all the components of ABA signalling except $\mathrm{ABA}$ receptors ${ }^{24}$ can complement Ppsnrk2 mutants. We propose that the ABA/ARK/ subclass III SnRK2 signalling module is an ancestral feature that enabled the colonization of land by simple plants, which subsequently recruited $\mathrm{ABA}$ to modulate the dehydration response before the separation of bryophytes and vascular plants. Subsequently, vascular plants further developed a novel subclass I SnRK2 signalling module for osmostress tolerance independent of the ABA-ARK system.

\section{Results}

Role of SnRK2 in ABA- and osmostress responses of $P$. patens. We established Ppsnrk2 null mutants of $P$. patens using sequential gene targeting of the Ppsnrk2a (Ppost1) plant (SKO) ${ }^{14}$ to generate the Ppsnrk2a/b double knockout (DKO), the Ppsnrk2a/b/c triple knockout (TKO), and two independent Ppsnrk2a/b/c/d quadruple knockouts (QKOs; line \#3 and \#7) (Supplementary Fig. 1a). The disruption of the four $P p S n R K 2$ genes through homologous recombination in QKO plants was confirmed by PCR (Supplementary Figs. 1b and 1c) and Southern blot analysis (Supplementary Fig. 1d). Null expression of PpSnRK2 genes in QKO (line \#7) was confirmed by RNA-seq analysis (Supplementary Fig. 1e).

Previously we identified a $40 \mathrm{kDa}$ kinase from $P$. patens protonemata activated by ABA and osmostress ${ }^{20,23}$. To confirm the identity of the kinase activity, proteins extracted from ABAor mannitol-treated protonemata of wild-type and Ppsnrk2 disruptants were subjected to the in-gel kinase assay (Figs. 1a, b). A decrease of kinase activity correlated with the number of disrupted genes, QKO plants showing little kinase activity after treatments. These results strongly support identification of the $40 \mathrm{kDa}$ kinases as PpSnRK2s. ABA responses of $P$. patens protonemata were also adversely affected in the SnRK2 disruptants (Supplementary Fig. 2a). Growth of wild-type protonemata is effectively repressed by ABA treatment, with structural changes leading to the formation of thick-walled spherical brood cells 25,26 . Protonemal growth of wild-type and Ppsnrk2 SKO, DKO, and TKO plants was inhibited on medium containing $10 \mu \mathrm{M}$ ABA, but QKO plants showed little growth inhibition, similar to the response of the ABA-insensitive ark mutant, which lacks functional $\mathrm{ARK}^{23}$. Even with $\mathrm{ABA}$ concentration increased to $100 \mu \mathrm{M}$, few brood cells were observed in QKO plants (Fig. 1c). P. patens protonemata are susceptible to freezing temperatures, but ABA pre-treatment markedly enhances freezing tolerance ${ }^{27}$. All plants except QKO mutants showed ABA-induced freezing tolerance comparable with that of wild-type (Supplementary Fig. 2b). Exogenous ABA also induces desiccation tolerance of $P$. patens protonemata ${ }^{17,18,28}$, and we found that ABA-treated wild-type protonemata could survive desiccation in an atmosphere of $75 \%$ relative humidity $(\mathrm{RH})(-39$ $\mathrm{MPa})^{17}$ for $24 \mathrm{~h}$. By contrast, ABA-treated QKO plants and the ark mutant could not (Supplementary Fig. 2c).

Hydrophilic late embryogenesis abundant (LEA) proteins are associated with desiccation tolerance of plant cells ${ }^{20,29}$. We confirmed the lack of expression of several LEA genes in QKO plants in response to ABA by RNA gel blot analysis (Fig. 1d). We further investigated the accumulation of boiling-soluble proteins, which is a feature of hydrophilic LEA proteins ${ }^{15}$. Exogenous ABA and mannitol-induced osmostress caused accumulation of boiling-soluble proteins in wild-type, but not in QKO plants (Fig. 1e). Immunoblot analysis using a specific antibody to 17B9 (Pp3c23_13700V3.1), a LEA-like protein associated with the osmostress response ${ }^{30}$ confirmed this (Fig. 1f). We then evaluated the osmostress tolerance of QKO plants. We dehydrated protonemal cells in an atmosphere of $97 \% \mathrm{RH}$ (corresponding to $-4 \mathrm{MPa}$ water potential) for 50,75 , and $100 \mathrm{~h}$ (Fig. 1g). As previously reported, this treatment reduces water content of the protonemata to about $60(50 \mathrm{~h}), 45(75 \mathrm{~h})$, and $40 \%(100 \mathrm{~h})$, respectively ${ }^{17}$. These protonemata were rehydrated and then recovered on normal medium. Both QKO plants and the ark mutant survived dehydration for $50 \mathrm{~h}$ like wild-type plants, suggesting that PpSnRK2s and ARK are not essential for mild (60\% water content) dehydration stress-tolerance. By contrast, whereas wild-type plants survived $75-100 \mathrm{~h}$ of dehydration, the QKO plants and the ark mutant did not. QKO plants were also hypersensitive to osmotic stress. Protonemata of QKO as well as 
a

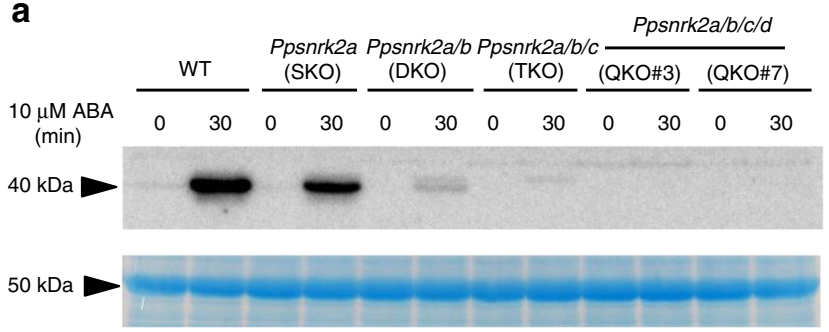

C

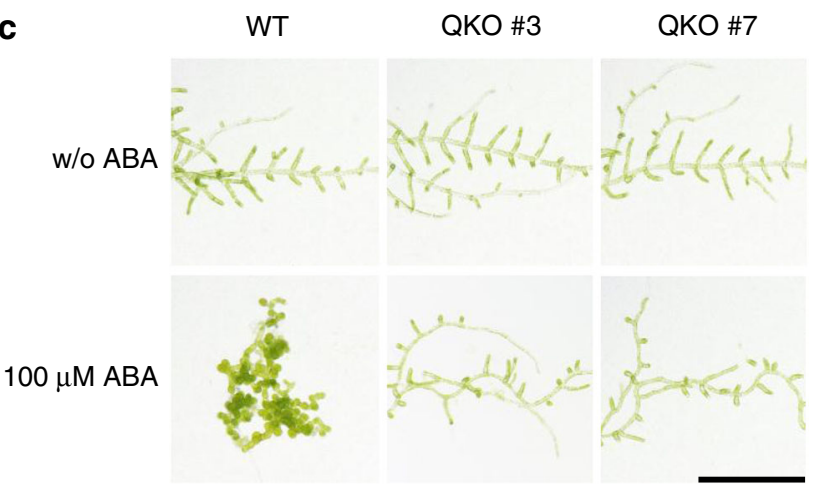

e

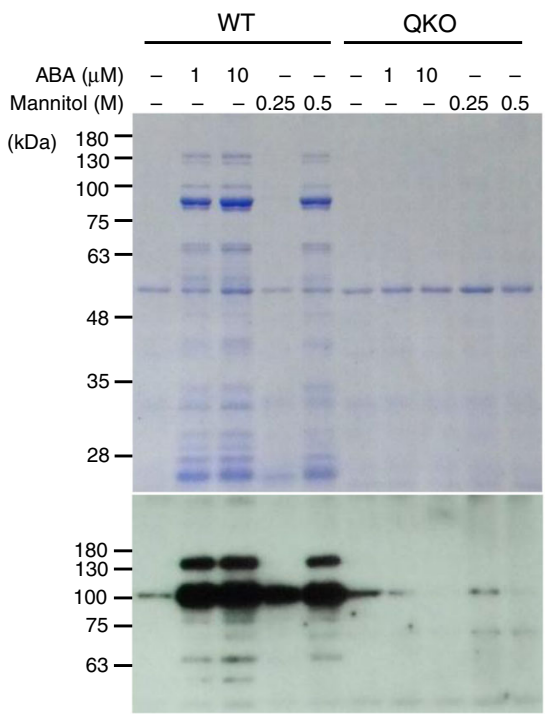

h

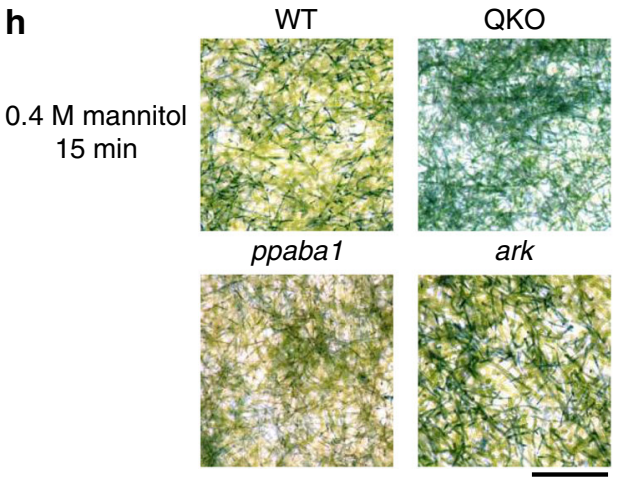

b

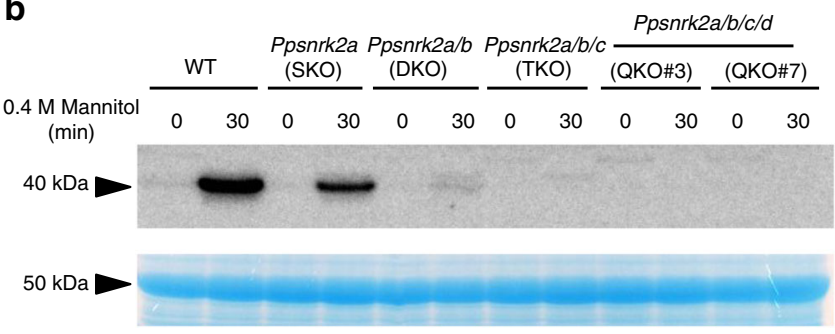

d

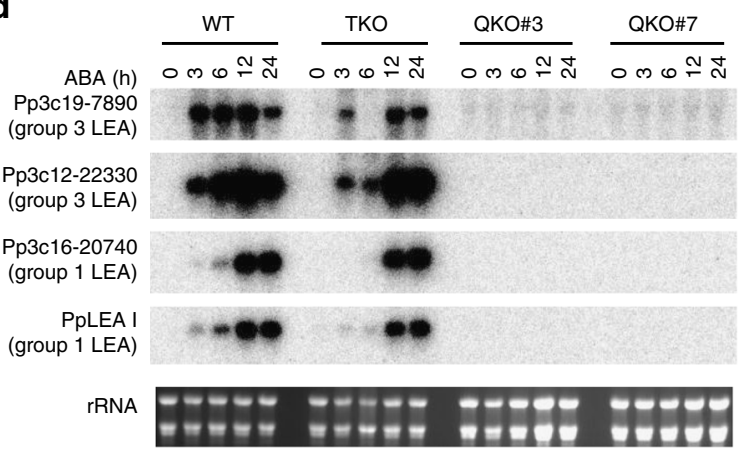

g
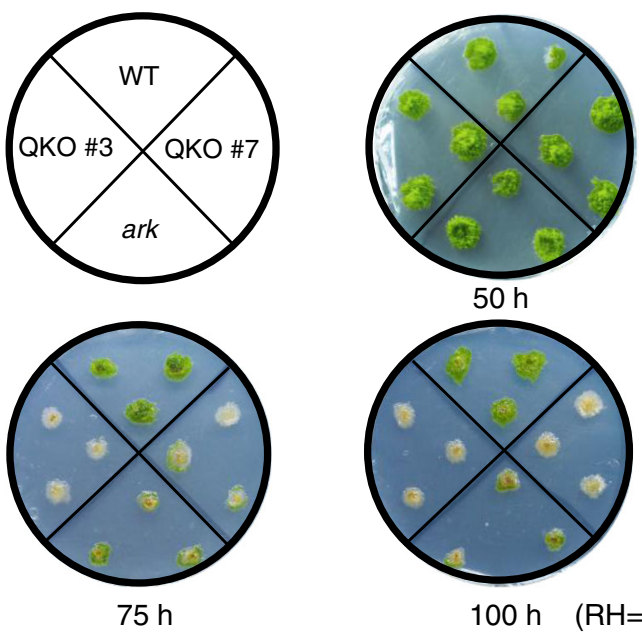

$100 \mathrm{~h} \quad(\mathrm{RH}=97 \%)$

i

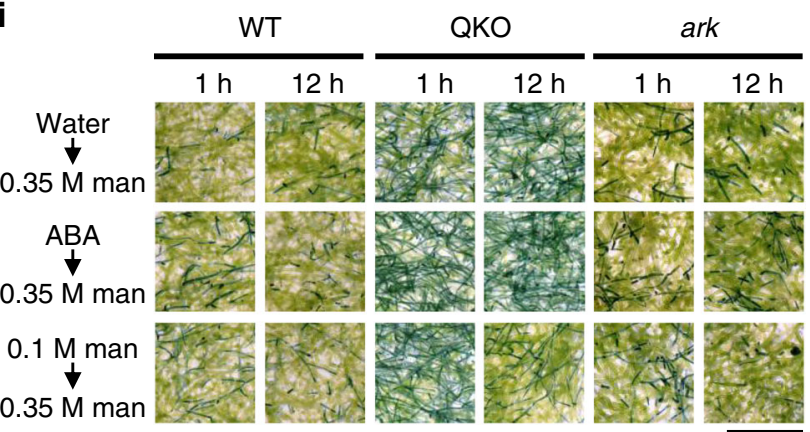

cells of the QKO plants did not survive this treatment. We also compared osmoshock tolerance of QKO with both ABA-deficient transgenic $P$. patens (ppaba1) $)^{30}$ and the ark mutant. The ABAdeficient mutant and to a lesser extent the ark mutant was tolerant to this treatment; however, QKO was not. This suggests

that an additional ABA- and ARK- independent SnRK2 function the ark mutant could not survive after culture on medium containing $1 \mathrm{M}$ mannitol (-2.5 MPa) (Supplementary Fig. 2d). Furthermore, when wild-type protonemata were subjected to osmoshock by exposure to a moderate concentration of $0.4 \mathrm{M}$ mannitol solution for $15 \mathrm{~min}$, the majority of cells survived, as judged by Evans blue staining (Fig. 1h), whereas most protonemal 
Fig. 1 Essential role of PpSnRK2 in ABA responses and osmostress tolerance. $\mathbf{a}$, b Activity of kinases from ABA-treated (a) or osmostress-treated (b) protonemata of wild-type (WT) and Ppsnrk2 disruptants. Total soluble proteins extracted from protonemata treated with $10 \mu \mathrm{M}$ ABA or with $0.4 \mathrm{M}$ mannitol for indicated periods were subjected to in-gel phosphorylation assay using histone IIIS as a substrate. The bottom panel shows Coomassie Brilliant Blue (CBB) staining of the protein samples to show equal loading. c Protonemal growth of WT and the Ppsnrk2 QKO plants with or without $100 \mu \mathrm{M}$ ABA. d RNA gel blot analysis of ABA-inducible LEA gene expressions of WT, Ppsnrk2 TKO, and QKO. Protonemata were treated with $10 \mu M$ ABA for indicated periods, and total RNA was used for the analysis. The bottom panel shows ethidium bromide staining of rRNA to show equal loading. e, $\mathbf{f}$ Accumulation of $\mathbf{e}$ boiling soluble proteins and $\mathbf{f}$ a LEA (17B9) protein in protonemata of WT or QKO plants treated with ABA or mannitol. Protonemata were treated with or without the indicated concentration of $A B A$ or mannitol for $1 \mathrm{~d}$ prior to protein extraction. e Extracted proteins were boiled, and the supernatant (boiling soluble proteins) was separated by SDS-PAGE and stained by CBB. $\mathbf{f}$ Extracted proteins were separated by SDS-PAGE, transferred onto a nylon membrane, and reacted with anti-17B9 antibody. $\mathbf{g}$ Desiccation tolerance of Ppsnrk2 QKO plants. The ABA-insensitive mutant ark was used as a control. Protonemata were dehydrated in an atmosphere of relative humidity of $97 \%$ for indicated periods. The dehydrated tissues were hydrated in water and grown on normal medium for two weeks. h Comparison of osmoshock tolerance among ABA mutants of $P$. patens. Protonemata of WT, QKO, ABA-deficient Ppaba1, and ark were treated with $0.4 \mathrm{M}$ mannitol solution for $15 \mathrm{~min}$, then stained by $0.5 \%$ Evans Blue. i Protonemata of WT, QKO, and ark were subjected to an acclimation assay. One-week-old protonemata were treated with $0.1 \mathrm{M}$ mannitol solution or $10 \mu \mathrm{M} \mathrm{ABA}$ for $1 \mathrm{~h}$ or $12 \mathrm{~h}$, then subjected to osmoshock treatment with $0.35 \mathrm{M}$ mannitol for $20 \mathrm{~min}$ and stained with $0.5 \%$ Evans Blue. Scale bars, $0.5 \mathrm{~cm}$ in $\mathbf{c}, \mathbf{h}, \mathbf{i}$

also enables wild-type cells to withstand osmoshock. It is possible that even without osmostress PpSnRK2 might be slightly activated through unknown upstream kinase(s) or by autophosphorylation. Nevertheless, QKO protonemata were susceptible even to mild osmoshock imposed by $0.35 \mathrm{M}$ mannitol; however, if pretreated with a non-detrimental osmostress (0.1 M mannitol) for $12 \mathrm{~h}$, they acquired tolerance to $0.35 \mathrm{M}$ mannitol (Fig. 1i). In contrast, ABA-pretreatment did not recover the osmotolerance of QKO protonemata, indicating that osmostress acclimation of $P$. patens involves an ABA- and SnRK2-independent system. Analysis of Ppsnrk2a/b/d TKO plants showed these to have ABA sensitivity comparable with wild-type (Supplementary Fig. 2e). Moreover, introduction of $P p S n R K 2 A$ or $-D$ sequences under the constitutive actin promoter was sufficient to complement the QKO mutant phenotype, restoring ABA sensitivity to protonemata (Supplementary Fig. 2f). This demonstrates that $P p S n R K 2$ genes are functionally redundant and play a major role in protonemal ABA signalling. Our data clearly demonstrated the essential role of SnRK2 in ABA signalling as well as in dehydration tolerance of the moss protonemata.

SnRK2 in global gene expression and protein phosphorylation. To investigate the effect of SnRK2 disruption on global gene expression we used RNA-seq analysis of wild-type and QKO plants treated with $10 \mu \mathrm{M}$ ABA $(12 \mathrm{~h})$ or subjected to osmostress with $0.4 \mathrm{M}$ mannitol (12 h) (Supplementary Data 1 ). The RNAseq data were verified by $\mathrm{qPCR}$ analysis of the response of signature $\mathrm{ABA} /$ stress-response genes (Supplementary Fig. 3). In wild-type plants, 16,924 genes were represented by more than five mapped reads per transcript. Of these 2187 were significantly $(>2$ fold with a false discovery rate with $P$ values less than 0.05 ) upregulated and 2880 were down-regulated by ABA treatment. Osmotic stress resulted in 1028 genes being significantly upregulated and 1595 down-regulated (Supplementary Fig. 4a). The majority of osmostress-responsive genes overlapped with ABAresponsive genes, suggesting that osmostress-induced ABA accumulation is responsible for these expression patterns. The number of ABA-responsive genes was greatly reduced in QKO plants whereas that of osmostress-responsive genes was relatively normal (Fig. 2a). Principal component analysis of gene expression profiles in QKO plants indicated that the effect of PpSnRK2 disruption appeared most prominently in the ABA response (Fig. 2b), as was also indicated by scatter plots of gene expression of QKO vs wild-type after ABA treatment $\left(R^{2}=0.0378\right)$ compared to that of osmostress treatment $\left(R^{2}=0.6108\right)$ (Fig. $\left.2 \mathrm{c}\right)$. We found a significant number of genes that were affected only by osmostress in wild-type (Supplementary Fig. 4a). Interestingly, expression of these genes was scarcely affected by disruption of
SnRK2. This cohort of ABA- and SnRK2-independent osmostress responsive genes represent candidate components of the osmostress acclimation process. It is noteworthy that RNA-seq analysis also demonstrated that the expression of many genes, including LEA (Supplementary Fig. 4b), were more drastically reduced in the QKO mutant than in the wild-type, under normal conditions. This may partly explain the reason for the hypersensitivity of QKO plants following osmoshock (Fig. 1h).

To gain insights into the characteristics of SnRK2-regulated ABA-upregulated genes (SAGs), we performed hierarchical clustering and heatmap analysis using various public $P$. patens RNA-seq data ${ }^{21,31-33}$. The SAGs were also upregulated by typical dehydration-related stresses (Fig. 2d). Functional categorization of SAGs, by gene ontology term enrichment analysis revealed that SnRK2s are involved mainly in abiotic stress responses and energy metabolism (Fig. 2e). We compared our transcriptomic data with that of the ark mutant ${ }^{23}$ for ABA-responsive genes (Supplementary Fig. 4c). Most ARK-regulated genes (91\% for $\mathrm{ABA}$-induced and $77 \%$ for ABA-repressed) were included in SnRK2-regulated genes, indicating the major role of the ABA/ ARK/SnRK2 module in the regulation of ABA-responsive gene expression. To investigate the overlap between responses of SnRK2-regulated gene expression in P. patens and Arabidopsis, we analysed transcriptomic data for the Arabidopsis srk2dei mutant, which lacks subclass III SnRK2, deposited in ArrayExpress (E-MEXP-3713; ${ }^{34}$ ) (Supplementary Fig. 4d and Supplementary Data 2). We found that about $20 \%$ of Arabidopsis subclass III-regulated ABA responsive genes were orthologues of $P$. patens SnRK2-regulated, ABA-responsive genes. Gene ontology term enrichment analysis of the overlapping gene sets showed enrichment of stress responsive genes (Supplementary Fig. 4e), indicating SnRK2 function to be evolutionarily conserved in regulating stress responses of land plants. We also investigated genes with fewer than 5 mapped reads in any samples, which were removed from analyses described above. These genes also contained a significant number of ABA- and SnRK2-regulated genes such as LEA genes (Supplementary Figure 5a and Supplementary Data 3). Gene ontology term enrichment analysis of these genes resulted in a similar pattern with that of SAGs (Supplementary Figure 5b).

In addition to the RNA-seq analysis, we performed phosphoproteome analysis to investigate the effect of SnRK2 deletion on ABA-responsive protein phosphorylation ${ }^{34}$. We detected 3789 unique phosphopeptides in protonemal proteins prepared from wild-type and QKO treated with or without ABA. Of these, 760 showed significant differences between the wild-type and QKO, and principal component analysis demonstrated that disruption of PpSnRK2 greatly affected the phosphoproteome profiles (Fig. 3a). In wild-type plants, 498 phosphopeptides 
a

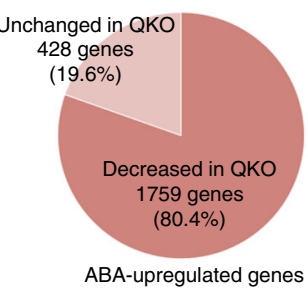

(2187)

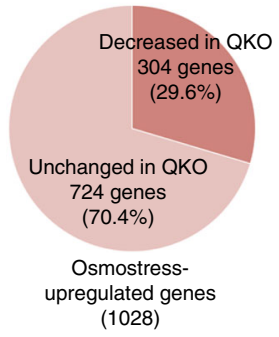

(1028)
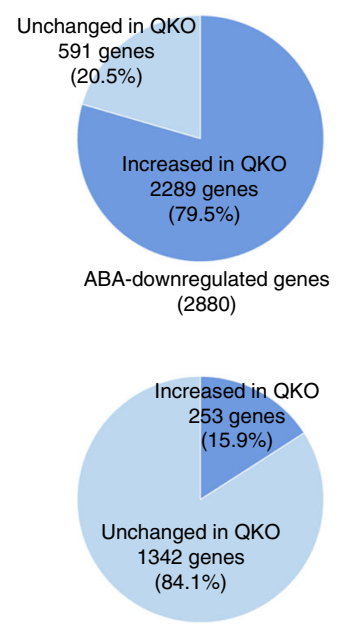

Osmostressdownregulated genes (1595)

d
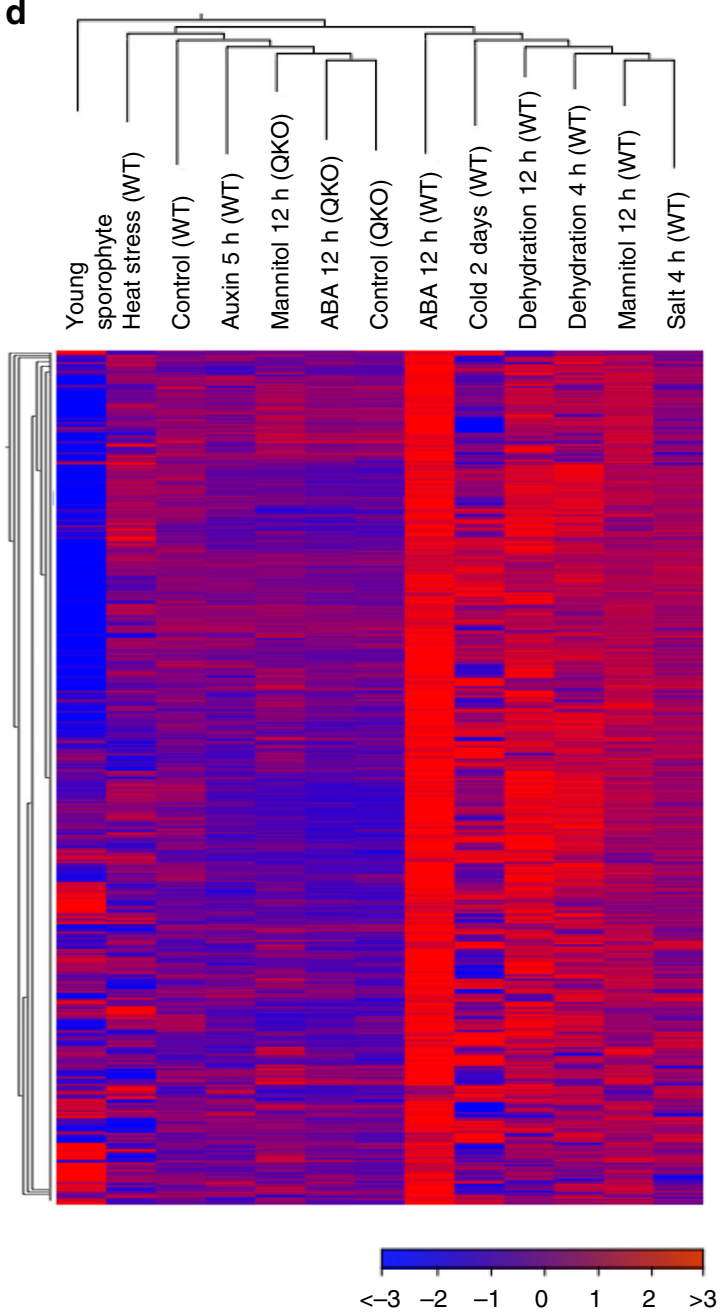

e
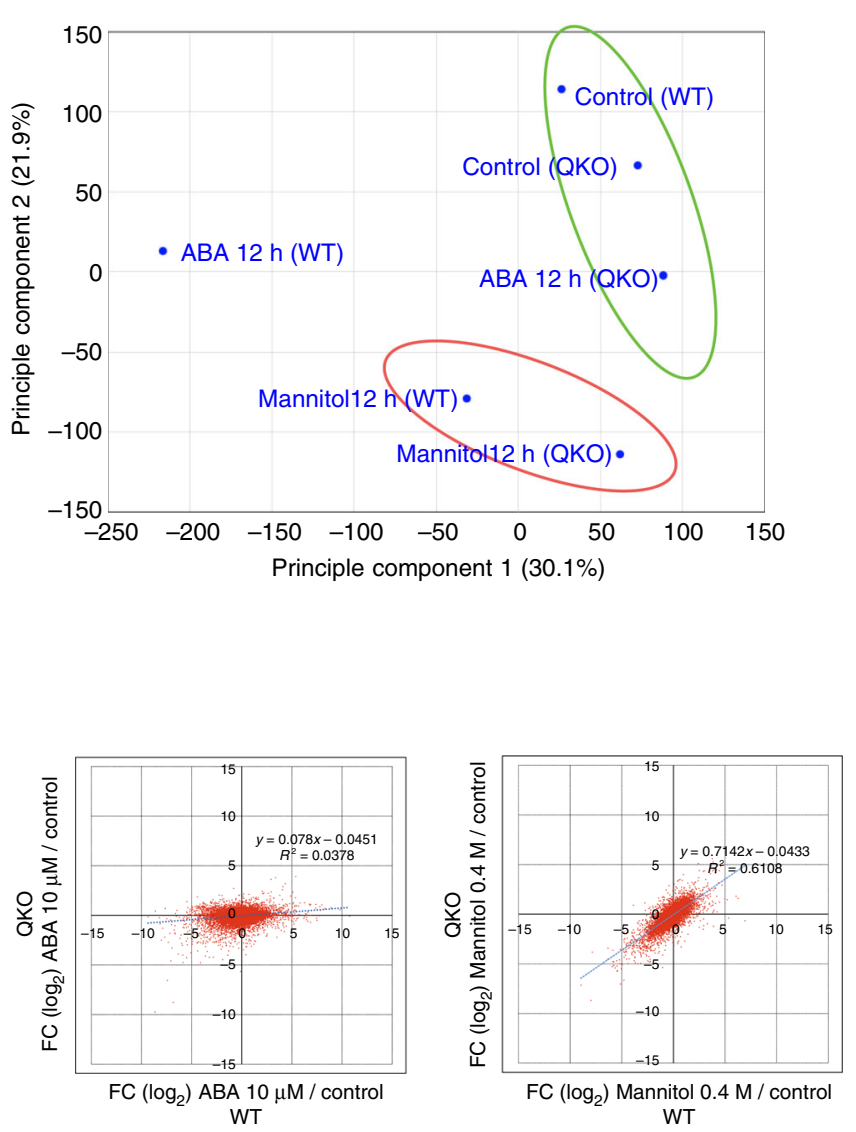

C

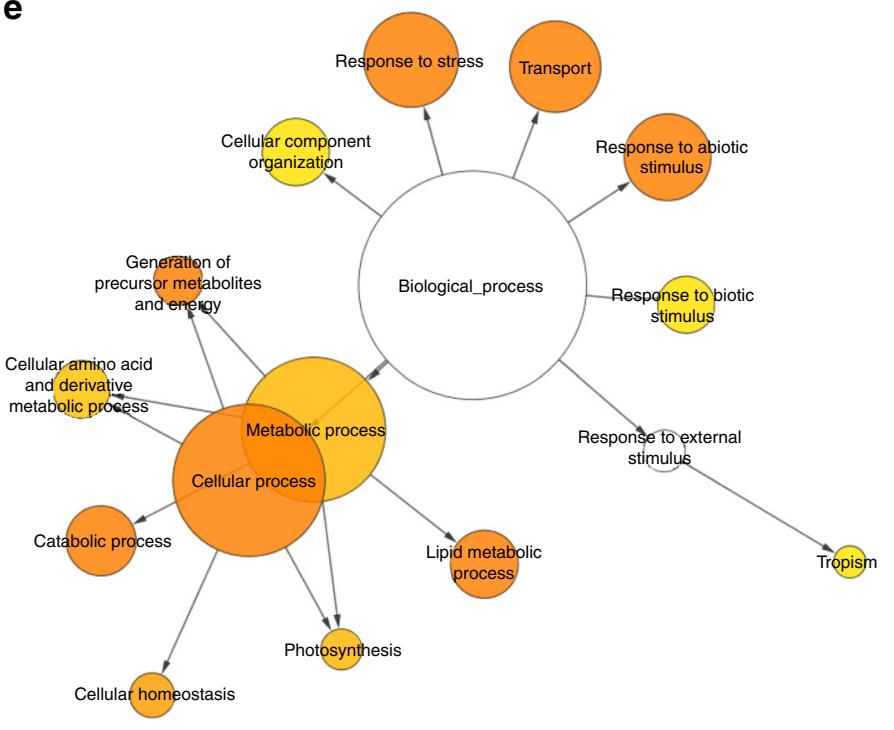

compared the ARK-regulated ABA-responsive phosphopeptides with the 138 ABA-responsive phosphopeptides regulated by PpSnRK2. The result showed that 24 proteins were commonly regulated by ARK and SnRK2, which included an ABAresponsive bZIP transcription factor (ABF)-related protein (Pp3c20_7230V3.2.p). Examples of these ABA-responsive phosphorylation profiles between wild-type and QKO are shown (Fig. 3c). An in vitro phosphorylation assay was performed to

showed a more than 3 -fold change in abundance after ABA treatment in at least two of the three biological replicates. In QKO plants, 138 (27.8\%) of the 498 ABA-responsive phosphopeptides (corresponding to 105 unique proteins) were lower in abundance ( $1 / 3$-fold compared to wild-type) (Fig. $3 \mathrm{~b}$ and Supplementary Data 4 and 5). Amagai et al. reported through the phosphoproteomic analysis of the ark mutant that ARK regulated $126(88.1 \%)$ of the 143 ABA-responsive phosphopeptides ${ }^{35}$. We

\section{b}


Fig. 2 Transcriptomic analysis of PpSnRK2 downstream factors. a Numbers of ABA-regulated genes and osmostress-regulated genes in wild-type (WT), and the numbers of genes affected in QKO plants. Genes with more than two-fold difference in the expression level between ABA-treated WT and QKO or mannitol-treated WT and QKO (false discovery rate (FDR) $\leq 0.05$ ) were categorized as Decreased or Increased in QKO. b Principal component analysis of gene expression profile in QKO plants. c Fold-changes (FC) (log2) after $12 \mathrm{~h}$ of (left) $10 \mu \mathrm{M} \mathrm{ABA}$ treatment or (right) $0.4 \mathrm{M}$ mannitol treatment of whole genes detected in the RNA-seq experiment were plotted. X-axis, WT; y-axis, QKO. d Hierarchical clustering and heatmap analysis were performed for the SnRK2-regulated ABA-inducible genes (SAGs). Gene expression values are represented as relative to the mean of all samples; blue represents lower expression, red represents higher expression (expression range, -3 to 3). Following high-throughput sequencing, data were obtained from the NCBI Sequence Read Archive, Auxin 5 h (SRR3350456), Dehydration 4 h (SRR1707403), Dehydration 12 h (SRR2225592), Heat stress (SRR790663), Salt 4h (SRR1707400), and Young sporophyte (SRR1553314). e Gene ontology term enrichment analysis was performed against SAGs. Functional enrichment analysis was performed for BLAST best-hit genes of SAGs, which were used to search Arabidopsis TAIR 10 proteins using the BiNGO plug-in in Cytoscape. Gene ontology Slim was used to summarize the network. The intensity of the color indicates significance of nodes based on corrected p-value (5\% FDR)

confirm whether SnRK2 could phosphorylate selected phosphopeptides identified in this study. We chose 3 phosphopeptides from the list of PpSnRK2-regulated phosphopeptides and one phosphopeptide which is not included in the list, then these peptides were subjected to in vitro phosphorylation assay by PpSnRK2B (Supplementary Figure 6 and Supplementary Data 6). Our data demonstrated that two peptides derived from a kinesin motor protein and a serine/threonine-protein kinase as well as a positive control peptide derived from a sucrose-phosphate synthase, which was shown to be phosphorylated by PpSnRK2B in vitro ${ }^{35}$, were strongly phosphorylated by PpSnRK2B, confirming that our phosphoproteomic data involved SnRK2 substrates. On the other hand, a PpSnRK2-regulated phosphopeptide from phototropin was only weakly phosphorylated to a similar extent with that of a phosphopeptide not regulated by PpSnRK2 derived from a calmodulin-binding transcription activator (CAMTA), suggesting that the phototropin may not be directly phosphorylated by PpSnRK2. It has been demonstrated in Arabidopsis that ABFs are SnRK2 substrates $34,36,37$ and four ABF transcription factors (ABF1, $\mathrm{ABF} 2, \mathrm{ABF} 3$, and $\mathrm{ABF} 4)$ are the master regulators for SnRK2-activated ABA-responsive transcription in vegetative tissues ${ }^{38,39}$. Our analysis in $P$. patens detected a conserved, ABA-responsive, SnRK2-dependent phosphopeptide NFGPSMNMDEFLK in a bZIP transcription factor, which is also found in the $\mathrm{C} 1$ domain of Arabidopsis ABFs and which is known to be phosphorylated by Arabidopsis subclass III SnRK2 in an ABA-dependent manner ${ }^{34}$. Our data suggest that ARK and SnRK2 together regulate ABA signalling through phosphorylation of $\mathrm{ABF}$ in $P$. patens and that the core $\mathrm{ABA}$ phosphosignalling is evolutionarily conserved between bryophytes and angiosperms. The Motif-X algorithm detected four motifs; [-R-X-X-pS-], [-pS-P-], [-pS- $-\mathrm{S}-\mathrm{S}-]$ and $[-\mathrm{S}-\mathrm{x}-\mathrm{pS}]$ from the phosphopeptides enhanced in response to ABA (Fig. $3 \mathrm{~b}$ and Supplementary Data 7$)$. The motif $[-\mathrm{R}-\mathrm{x}-\mathrm{x}-\mathrm{pS}-]$ was the only sequence common to both ABA-enhanced and SnRK2-dependent datasets. This motif was also identified as a phosphorylation target motif of Arabidopsis subclass III SnRK2 ${ }^{38,34}$, representing the evolutionarily conserved phosphorylation preference in subclass III SnRK2 in land plants. We also detected the [-pS-P-] motif in SnRK2-independent ABA-responsive phosphopeptides. This motif is a minimal mitogen-activated protein kinase (MAPK) target motif, suggesting an involvement of MAPK cascade in ABA signalling of the moss, as demonstrated in Arabidopsis ${ }^{34,39}$. The [-pS/pT-x-S-] and [-S-x-pS/pT-] motifs were not detected in previous phosphoproteome analyses of Arabidopsis SnRK2 or of Physcomitrella ARK, suggesting that these motifs are speciesspecific recognition motifs of SnRK2 in the moss and represent differences in ABA-responsive phosphosignaling between bryophytes and angiosperms ${ }^{35}$. It is noteworthy that there were only three overlaps of SnRK2-regulated proteins between $P$. patens and Arabidopsis ${ }^{34,39}$ \{AT3G13300/Pp3c15_3450V3.1.p (varicose); AT3G57150/Pp3c22_190V3.1.p (putative pseudouridine synthase NAP57); AT1G49720.1/ Pp3c20_7230V3.2.p (ABF1 abscisic acid responsive element-binding factor 1)\}. This observation may imply the differential evolution of ABA-responsive phosphosignalling downstream of SnRK2 after the separation of bryophytes and vascular plants, as suggested in the previous report ${ }^{35}$. Nevertheless, 360 peptides were phosphorylated in response to $\mathrm{ABA}$ in $\mathrm{QKO}$ plants, suggesting the presence of an alternative, SnRK2-independent, phosphorylation cascade(s) in response to $\mathrm{ABA}$.

Activation of SnRK2 via the ARK recognition motif. Previously, we showed that the Raf-like kinase ARK plays an essential role in ABA signalling in moss and that the ark mutant failed to activate the ABA-activated kinases ${ }^{23}$, which are shown to be PpSnRK2s in this study. In vitro, ARK phosphorylated Ser-165 and/or Ser-169 (SQPKS) within the activation loop of PpSnRK2B that elicits the kinase activity ${ }^{23}$. We generated transgenic plants over-expressing green fluorescent protein (GFP) fusion proteins of PpSnRK2B (PpSnRK2B-GFP) or the mutated protein in which two serine residues were substituted for alanine residues (PpSnRK2B-GFP(S165/169 A)) in a wild-type background. The ABA-induced kinase activity of the wild-type PpSnRK2B-GFP protein was abolished by Ala substitutions of Ser-165 and Ser-169 (S165/169 A) (Fig. 4a). We also performed functional complementation by the PpSnRK2B protein by a transient assay. Transcription from a wheat $E m$ promoter is activated by ABA in moss, and can be monitored by the transient assay of protonemal cells using particle bombardment of the $E m$ promoter fused to $\beta$ glucuronidase (GUS) reporter gene $(E m-G U S)^{40}$. ABA-responsive Em-GUS expression was abolished in the QKO protonemata (Fig. 4b). Co-introduction of the PpSnRK2B-GFP effector construct with Em-GUS into the QKO protonemata restored ABAresponsive Em-GUS expression; however, PpSnRK2B-GFP (S165) 169 A) completely failed to restore the ABA response. The core sequence $\underline{S} Q P K \underline{S}$ is evolutionarily conserved among subclass III SnRK2s, but it has been changed to $S[K / R / N / M] P K S$ in subclass I SnRK2s (Supplementary Fig. 7a). To confirm the importance of the conserved SQPKS motif for the subclass III SnRK2 activation, we introduced mutations into PpSnRK2D to change the consensus sequence to the subclass I-type and monitored activity using an endogenous ABA-responsive PpLEA1 promoter (PpLEA1-GUS $)^{41}$ (Fig. 4c). The single mutation of Gln to either Met or Asn significantly reduced the activation of PpLEA1-GUS. Mutation to the basic amino acids Arg or Lys (the most frequent amino acids in the subclass I motif) further decreased PpSnRK2D effector activity. We also tested the activation of Em-GUS by osmostress because PpLEA1-GUS did not show osmostress-dependent activation in this assay (Supplementary Fig. 7b). The mutated versions of PpSnRK2D all showed reduced activity in response to osmostress, comparable with the reductions in ABA-responsiveness (Fig. 4d). We further confirmed the importance of the SQPKS motif in vitro. PpSnRK2D with or without mutations fused to maltose-binding protein (MBP) and 
a

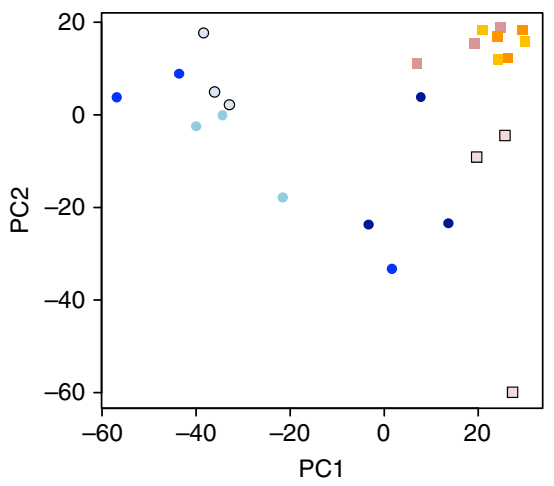

WT $\bigcirc 0 \mathrm{~min} \bullet 15 \mathrm{~min} \bullet 30 \mathrm{~min} \bullet 90 \mathrm{~min}$

QKO $\square 0 \mathrm{~min} \square 15 \mathrm{~min} \quad 30 \mathrm{~min} \square 90 \mathrm{~min}$ b

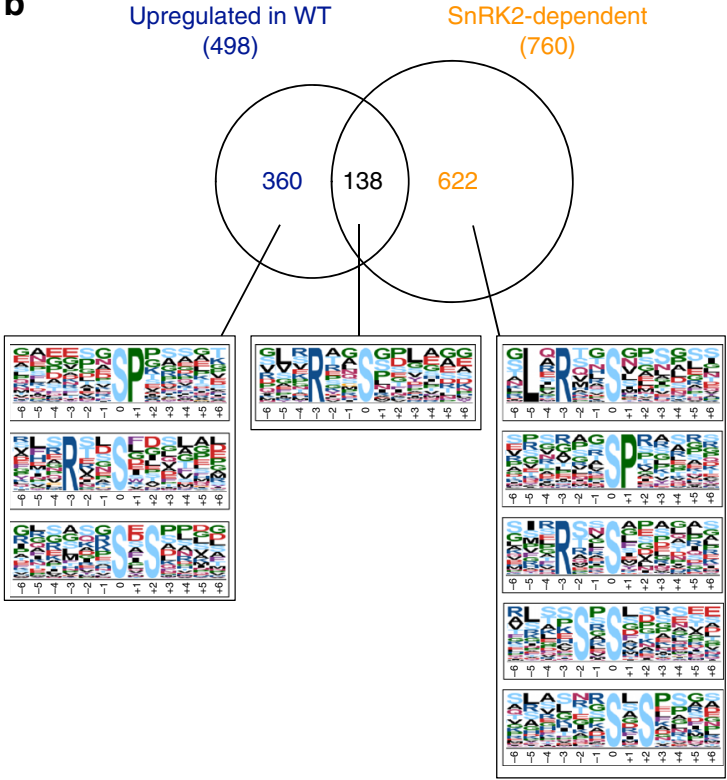

C
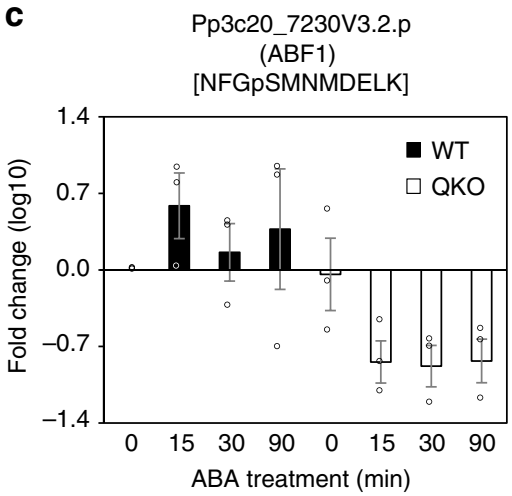

Pp3c5_11880V3.1.p (COR)

[RVEEPGYGYDQQTSEPTSLEpSGTQHSPKK]

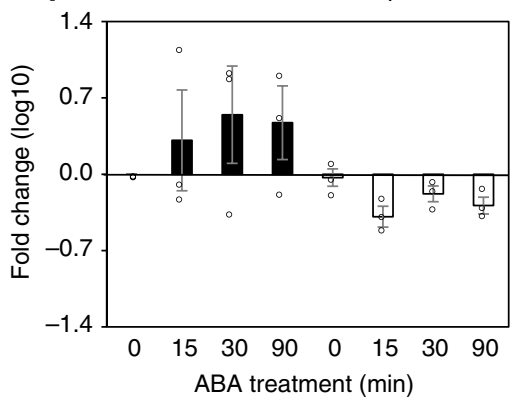

Pp3c27 6350V3.1.p

(MRF1)

[LVSVGpSAEVK]

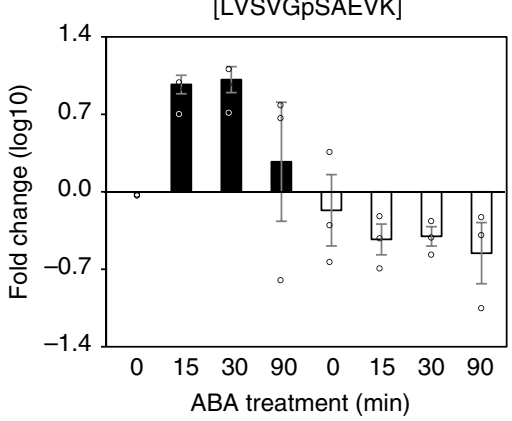

Pp3c22_17930V3.1.p (NRP1)

[DAGFGGLMpSTASDMFK]

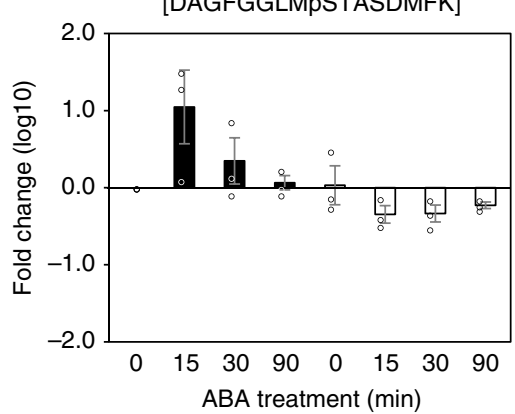

Fig. 3 Phosphoproteomic analysis of $P$. patens WT and Ppsnrk2 QKO plants. a Principal component analysis of quantitative data of phosphopeptides from wild-type (WT) and QKO plants under ABA treatment. b A Venn diagram of two phosphopeptide groups; one is the ABA-responsive (upregulated) group in WT, and the other is the SnRK2-dependent group. Phosphorylation motifs in those phosphopeptides were analyzed by the Motif-X algorithm. $\mathbf{c}$ Examples of ABA-responsive phosphopeptides in WT (solid) and QKO (empty). Pp3c20_7230V3.2.p; ABF transcription factor, Pp3c27_6350V3.1.p; MRF1 (MA3 domain-containing translation regulatory factor), Pp3c5_11880V3.1.p; COR (dehydrin), and Pp3c22_17930V3.1.p; NRP1 (nucleosome assembly protein 1). The relative peak areas to that of WT control $(0 \mathrm{~min})$ for respective phosphopeptides were calculated in each replicate using the quantitative data described in Supplementary Data 4 . The values are mean \pm S.E. $(n=3)$

the kinase domain of ARK fused to glutathione S-transferase (GST) were expressed in Escherichia coli, and affinity-purified proteins were subjected to in vitro phosphorylation assay. Although mutations of Gln to either Met or Asn did not show any significant effect on phosphorylation by ARK, mutation to the basic amino acids Arg or Lys significantly reduced the phosphorylation level by ARK (Supplementary Fig. 7c). Thus the evolutionarily conserved motif in the activation loop of subclass III SnRK2s is important for the ABA-dependent activation of the kinase through ARK. Since we observed partly different effects of the mutations between the in vitro phosphorylation assay and the in vivo functional assay, it is possible that unknown factor(s) such as a protein phosphatase is involved in the regulation of phosphorylation status of the motif. 
a
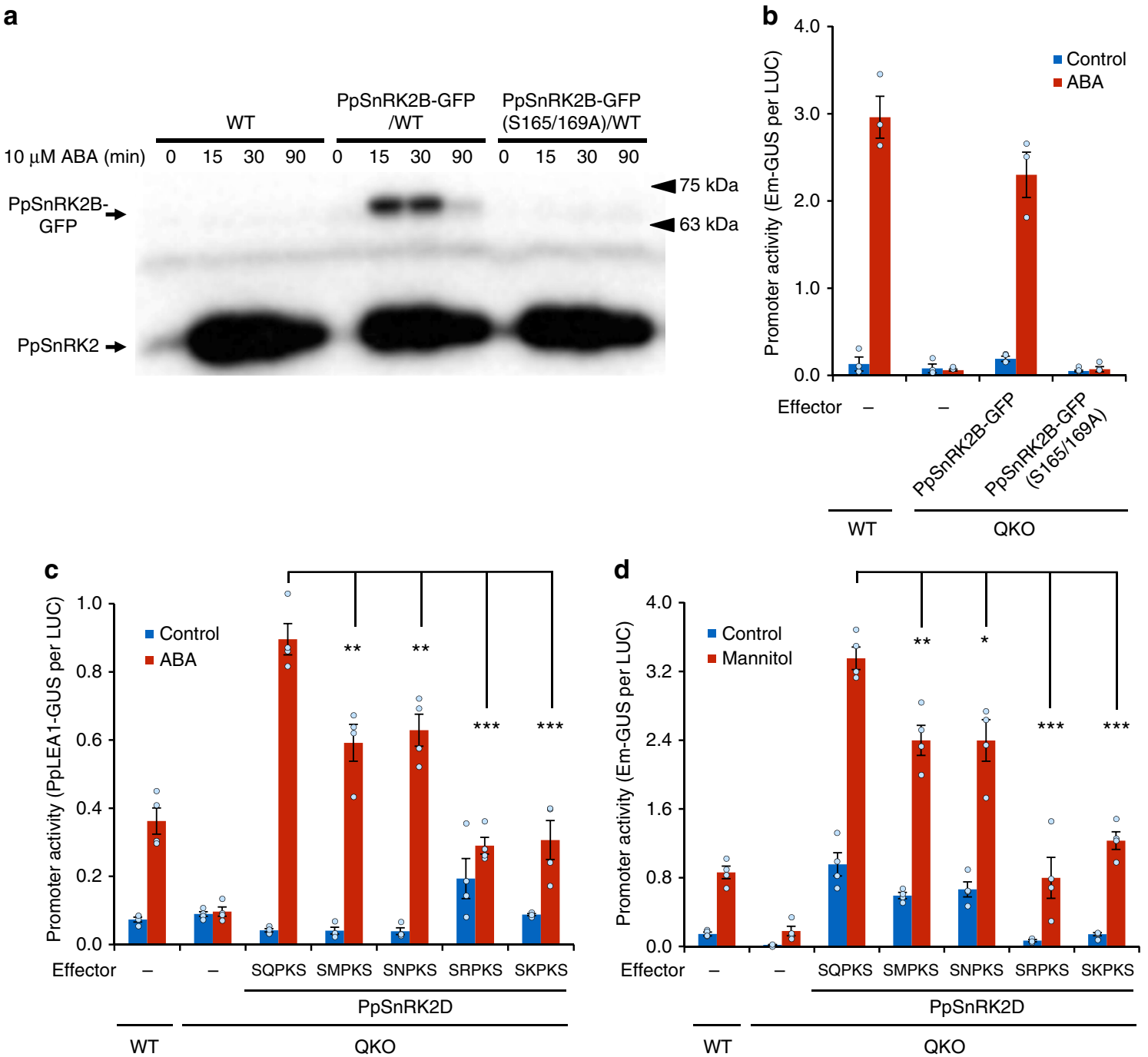

Fig. 4 ABA-activated SnRK2 activity of $P$. patens depends on the evolutionarily conserved ARK phosphorylation motif in the activation loop. a Protonemata of wild-type (WT) or transgenic plants expressing SnRK2B-GFP or mutated SnRK2-GFP (S165/169 A) under the control of the rice actin promoter were treated with or without $10 \mu \mathrm{M} \mathrm{ABA}$ for the indicated periods in minutes, then the protein extracts were subjected to in-gel kinase assay. Arrows indicate the position of recombinant SnRK2B (SnRK2-GFP) and endogenous SnRK2. b Constitutive rice actin promoter-driven SnRK2B-GFP or mutated SnRK2-GFP (S165/169 A) in the predicted phosphorylation sites was introduced into QKO protonemata with Em-GUS and Ubi-LUC by particle bombardment. Protonemal cells were incubated with or without $10 \mu \mathrm{M}$ ABA for 1 day, then subjected to GUS and LUC assays. Promoter activity of the Em promoter in WT and QKO in the absence of the effector construct is shown as controls. Levels of gene expression are represented by GUS per LUC ratio. Error bars indicate the standard error $(S E ; n=4)$. c, d PpSnRK2D or its mutant variants fused to PpSnRK2D gene native promoter were introduced into QKO protonemata with PpLEA1-GUS for $10 \mu \mathrm{M}$ ABA treatment for $1 \mathrm{~d}(\mathbf{c})$, or with Em-GUS for $0.4 \mathrm{M}$ mannitol treatment for $1 \mathrm{~d}$ (d). Promoter activity of promoters in WT and QKO in the absence of the effector construct are shown as controls. Levels of gene expression are represented by the GUS per LUC ratio. Error bars indicate the $\mathrm{SE}(n=4) .{ }^{\star} P<0.05,{ }^{\star \star} P<0.01,{ }^{\star \star \star} P<0.001$ compared with the PpSnRK2D effector of QKO with ABA

Evolutionarily conserved function of subclass III SnRK2. To investigate the evolutionarily conserved function of SnRK2 in land plants, we performed a complementation assay of $S n R K 2$ genes within moss and cross-species complementation using a transient assay of QKO protonemata (Fig. 5a). The failure of ABA-dependent activation of PpLEA1-GUS in the QKO plants was recovered by co-bombardment of each $P p S n R K 2$ gene driven by the $P p S n R K 2 D$ promoter. To evaluate whether SnRK2s from angiosperms could complement the function of Physcomitrella SnRK2, we used representative $S n R K 2$ genes of subclasses I (SnRK2.5), II (SnRK2.8), and III (SnRK2.6) from Arabidopsis as effectors in the transient assay under the control of the $P p S n R K 2 D$ gene promoter. As a result, Arabidopsis $S n R K 2$ genes of subclasses II and III but not subclass I restored ABA responsiveness to the QKO plants, as judged by the PpLEA1 promoter activity (Fig. 5a). We further tested if SnRK2 of non-land plants could rescue the function of PpSnRK2. Strikingly, the SnRK2 of the semiterrestrial alga $K$. nitens ${ }^{24}$ also rescued the ABA-induced expression of PpLEA1-GUS in QKO (Fig. 5a). We further performed the transient assay using the ark null-mutant background (Supplementary Fig. 8a). Arabidopsis SnRK2.6 and 2.8 could not induce PpLEA1-GUS expression by ABA in the ark background, indicating that Arabidopsis subclass III SnRK2s also require ARK for activation by ABA in the moss. Our data suggest that ancestral $S n R K 2$ genes capable of functioning in ABA signalling emerged prior to the evolution of land plants.

In addition, we investigated the kinase function in response to osmostress (Fig. 5b). Arabidopsis subclasses II and III induced Em-GUS expression in response to osmostress, whereas subclass I SnRK2.5 again failed to activate it. The osmostress activation of 
a

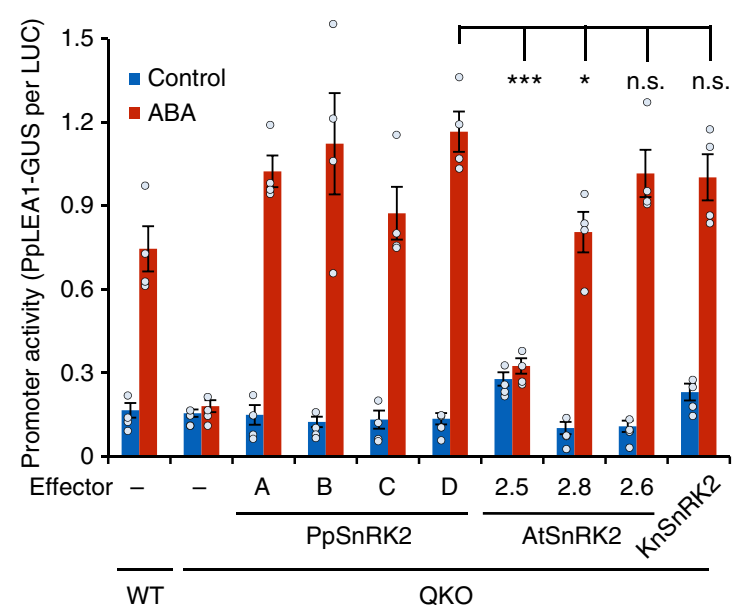

b

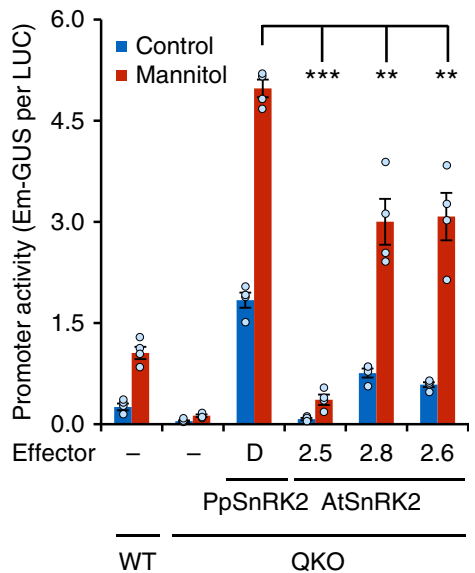

C
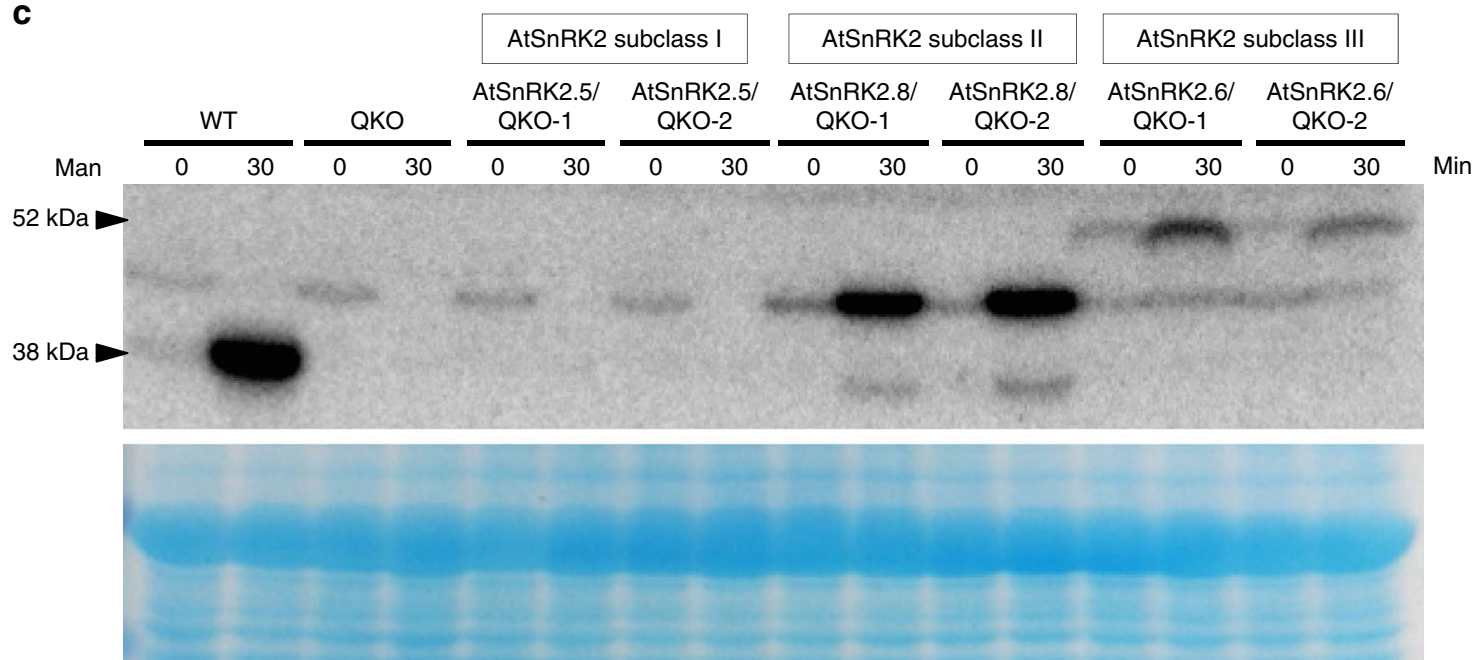

d
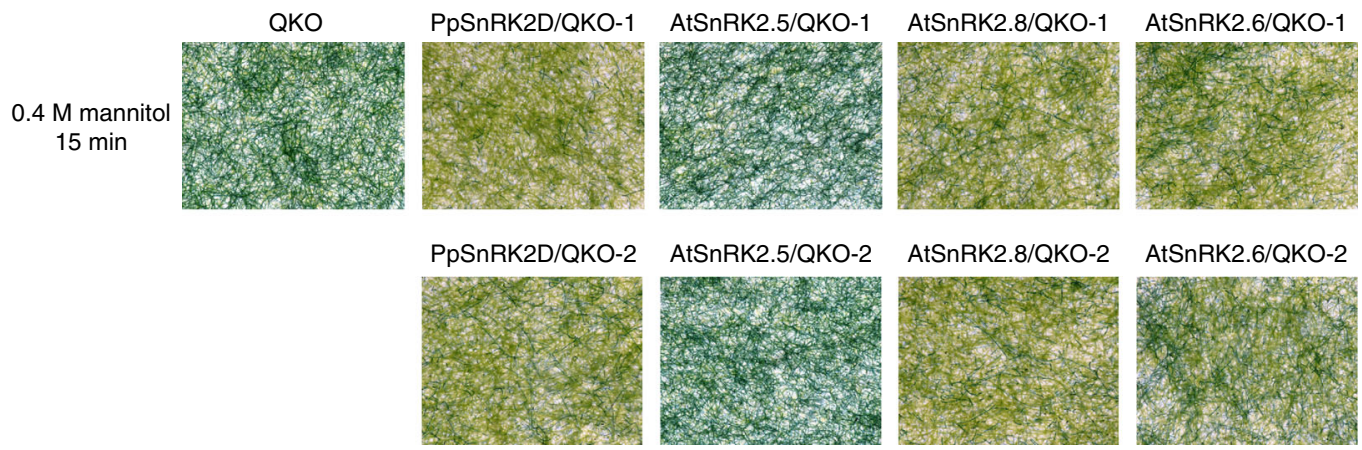

AtSnRK2.6/QKO-2

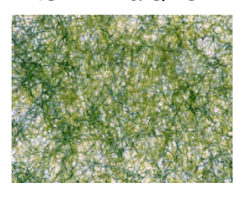

Fig. 5 Evolutionarily conserved function of subclass III SnRK2 in ABA- and osmostress signaling. a SnRK2 genes from K. nitens, P. patens and Arabidopsis were fused to the PPSnRK2D gene promoter and introduced into QKO protonemata with PpLEAT-GUS by particle bombardment. After bombardment protonemata were treated with or without $10 \mu \mathrm{M}$ ABA for 1d. $\mathbf{b}$ SnRK2 genes from Arabidopsis were fused to the PpSnRK2D gene promoter and introduced into QKO protonemata with Em-GUS and Ubi-LUC by particle bombardment. After bombardment protonemata were treated with or without $0.4 \mathrm{M}$ mannitol for 1d. Levels of gene expression are represented by GUS per LUC ratio. Error bars indicate the SE $(n=4)$. ${ }^{\star} P<0.05$, ${ }^{\star \star} P<0.01$, ${ }^{\star \star \star} P<0.001$ compared with the PpSnRK2D effector of QKO with ABA (a) or with mannitol (b). n.s., not significant. c, d Two independent transgenic $P$. patens plants that stably express Arabidopsis subclass I SnRK2.5, Subclass II SnRK2.8, or subclass III SnRK2.6 under PpSnRK2D gene promoter in QKO background were treated with $0.4 \mathrm{M}$ mannitol for $30 \mathrm{~min}$ and subjected to the in-gel phosphorylation assay (c), or treated by $0.4 \mathrm{M}$ mannitol solution for 15 min followed by Evans Blue staining to visualize the dead cells $(\mathbf{d})$. Scale bars, $1 \mathrm{~cm}$. WT, wild-type

Arabidopsis SnRK2s was confirmed in transgenic moss strains expressing Arabidopsis SnRK2s in the QKO background (Fig. 5c and Supplementary Fig. 8b). Arabidopsis SnRK2.8 and 2.6 but not Subclass I SnRK2s $(2.4,2.5$, and 2.10) were activated by osmostress, although SnRK2 proteins were expressed similarly in the transgenic plants (Supplementary Fig. 8c). Moreover,
Arabidopsis SnRK2.8 and 2.6 transformants restored the ABA sensitivity and osmotolerance of QKO mutant plants, whereas Subclass I SnRK2s did not (Fig. 5d and Supplementary Figs. 9a and $9 \mathrm{~b})$. Thus, these data suggested that the system for osmostress activation of SnRK2 subclasses II and III is evolutionarily conserved between the bryophyte and Arabidopsis, 
whereas the bryophyte does not possess the necessary upstream and/or downstream factors for subclass I SnRK2s to function in osmostress responses. Our result also agreed well with the previous report that the activation mechanism of Arabidopsis subclass III SnRK2 by osmostress is different from that of subclass $\mathrm{I}^{42}$

\section{Discussion}

Our results show the fundamental role of SnRK2 in ABA signalling as well as in osmostress and dehydration tolerance (including desiccation) of $P$. patens, an extant member of a basal land plant lineage. Furthermore, we demonstrated that even an algal SnRK2 from the evolutionarily distant charophyte, $K$. nitens, is functionally equivalent to that of land plants in executing $\mathrm{ABA}$ signalling, clearly identifying that the origins of SnRK2-mediated responses predate the evolution of land plants. Previous reports suggest that subclass III SnRK2 is the prototype of the SnRK2 family and that SnRK2 subclasses I and II evolved after separation of bryophytes and vascular plants, with the former representing the most recent form ${ }^{6,43}$. Taking advantage of the increased genome information from evolutionarily distant plant species such as charophycean algae and gymnosperms, we provided further insights into $S n R K 2$ gene family evolution by a survey of SnRK2 genes from published genome sequences and expressed sequence tags from algae and land plants (Supplementary Figs. 10-14). All the charophycean algae investigated so far (but none of the chlorophytes) possess only a single SnRK2 gene that belongs to subclass III (Supplementary Fig. 10). In bryophytes, the mosses Sphagnum fallax and Physcomitrella patens, and the liverwort Marchantia polymorpha, also have only subclass III SnRK2 genes (Supplementary Fig. 11). The other subclasses of SnRK2 are detected only in vascular plants (Supplementary Figs. 12 and 13). Thus it is likely that the precursor of the SnRK2 genes (subclass III-type) arose before the evolution of land plants, and that gene duplication and subfunctionalisation (the emergence of subclasses I and II) occurred after the separation of bryophytes and vascular plants (Supplementary Fig. 14). The land plants are monophyletic in origin, indicating descent from a single successful colonisation of the terrestrial environment by an aquatic ancestor within the charophyte algae approximately 500 million years ago ${ }^{44}$. Phylogenomic analyses have supported the identification of either the Charales ${ }^{45}$ or the Zygnematales ${ }^{46}$ as sister to the land plants, with the argument provided by Wickett et al. ${ }^{46}$ favouring the Zygnematales currently the most convincing, based as it is on phylogenetic reconstruction from the extensive sequence data generated in the 1000 plants programme. A number of extant charophyte species occupy semiterrestrial habitats, supporting the hypothesis that the successful colonisation of land derived from algal adaptations that enabled these anatomically simple algae to survive the challenging environmental conditions encountered on land ${ }^{47-49}$. Bryophytes are thought to be the most ancient extant land plant lineages, and the ability to tolerate dehydration must have been a key trait enabling successful colonization of land, and in land plants this trait is regulated by ABA. Significantly, although charophyte algae do not respond to $\mathrm{ABA}^{50}$, the Klebsormidium genome shares with the land plants all the core genes of ABA signalling with the exception of $\mathrm{ABA}$ receptors 24 , and our observation that the Klebsormidium SnRK2 ortholog can complement the P. patens QKO mutant in transducing an ABA signal supports the view that the recruitment of $\mathrm{ABA}$ to regulate a pre-existing ARK-SnRK2dependent dehydration response was a major adaptation enabling the successful colonisation of terrestrial environments. It is evident from the transient assay that orthologs of $\mathrm{ABA}$ receptors (PYR/PYL/RCAR) in $P$. patens function in ABA signalling upstream of SnRK2 since their introduction into wild-type cells enhanced the ABA response of the PpLEA1 promoter but no effect was observed when introduced into QKO (Supplementary Fig. 15). Although the activation of Arabidopsis SnRK2 is regulated through $\mathrm{ABA}$-dependent interaction between $\mathrm{ABA}$ receptors and PP2CA, P. patens PP2CA seems to act downstream of SnRK2 ${ }^{20}$. Recent phosphoproteomic analysis of $P$. patens demonstrated that SnRK2 was phosphorylated in an ABAdependent manner in the absence of PP2CA 35 , indicating the existence of an additional ABA-signal transduction pathway independent of the PYL/PP2CA interaction. These observations suggest an ancestral form of ABA signalling, in which ABAdependent activation of SnRK2 is mediated through ARK. ARK belongs to the $\mathrm{B} 3$ mitogen-activated protein kinase kinase kinase (MAPKKK) family, which can be divided into three subgroups (ARK-like, CONSTITUTIVE TRIPLE RESPONSE1-like, and ENHANCED DISEASE RESISTANCE-like), but ARK-like B3MAPKKK was lost during land plant evolution ${ }^{21}$, probably by acquiring a new mechanism to regulate subclass III SnRK2 by PP2CA through the PYL-ABA interaction. Emergence of a novel subclass I SnRK2 signalling module after the separation of bryophytes and vascular plants might have enabled vascular plants to develop new systems to cope with osmostress conditions independent of $\mathrm{ABA} / \mathrm{PP} 2 \mathrm{CA}$ regulation.

\section{Methods}

Plant materials and growth condition. The Gransden wild-type strain of Physcomitrella patens ssp. patens was used as the wild-type strain. Protonemal tissue was grown on cellophane-layered BCDAT medium (1.84 mM $\mathrm{KH}_{2} \mathrm{PO}_{4}$ [pH 6.5], $10 \mathrm{mM} \mathrm{KNO}{ }_{3}, 1 \mathrm{mM} \mathrm{MgSO}, 1 \mathrm{mM} \mathrm{CaCl}_{2}, 45 \mu \mathrm{M} \mathrm{FeSO}_{4}, 10 \mu \mathrm{M} \mathrm{H}_{3} \mathrm{BO}_{3}, 2 \mu \mathrm{M}$ $\mathrm{MnCl}_{2}, 0.22 \mu \mathrm{M} \mathrm{CuSO}_{4}, 0.23 \mu \mathrm{M} \mathrm{CoCl}_{2}, 0.19 \mu \mathrm{M} \mathrm{ZnSO}_{4}, 0.1 \mu \mathrm{M} \mathrm{Na}_{2} \mathrm{MoO}_{4}$, $0.17 \mu \mathrm{M} \mathrm{KI}, 5 \mathrm{mM}$ ammonium tartrate, $0.8 \%$ agar) at $25^{\circ} \mathrm{C}$ under continuous light (50-80 $\left.\mu \mathrm{mol} \mathrm{m}^{-2} \mathrm{~s}^{-1}\right)$. P. patens protonemata grown on a cellophane sheet were randomly subjected to experiments.

Gene targeting of PpSnRK2 genes. To create the targeting constructs for $P p S n R K 2 B$ (Pp3c6_16600), PpSnRK2C (Pp3c6_11090), and PpSnRK2D (Pp3c5_17150), regions of about $2 \mathrm{Kbp}$ upstream and downstream of the open reading frames were amplified by PCR using KOD Plus Neo (TOYOBO). The amplified fragments were verified by sequencing and cloned into p35S-loxP-BSD (PpSnRK2B), p35S-loxP-HPT (PpSnRK2C), or p35S-loxP-Zeo (PpSnRK2D). p35S loxP-BSD and p35S-loxP-Zeo were a generous gift from Mitsuyasu Hasebe, National Institute for Basic Biology, Okazaki, Japan. The resulting plasmids were used for generating $P p S n R K 2$ disruption mutants. The $P p O S T 1 / S n R K 2 A$ $\left(P p 3 c 5 \_21160\right)$ disruptant $(\mathrm{SKO})^{14}$ was used to establish a $p p s n r k 2 a / 2 b$ double disruptant (DKO) by transforming the PpSnRK2B targeting construct by polyethylene glycol (PEG)-mediated protoplast transformation ${ }^{51}$ with $30 \mu \mathrm{g} \mathrm{mL}^{-1}$ blasticidin $\mathrm{S}$ for the screening. The resulting $\mathrm{DKO}$ plant was used for generating the Ppsnrk2a/2b/2c disruptant (TKO) with $25 \mu \mathrm{g} \mathrm{mL}-1$ hygromycin for the screening, then the TKO plant was used for generating Ppsnrk2a/2b/2c/2d (QKO) with $50 \mu \mathrm{g} \mathrm{mL}^{-1}$ Zeocin. Disruption of SnRK2 genes was confirmed by genomic PCR using the specific primer sets (Supplementary Fig. 1b). The DKO plant was transformed with the $P p S n R K 2 D$ targeting construct for generating the Ppsnrk2a/2b/2d (ABD-TKO)

For complementation of $S n R K 2$ genes, the hygromycin-resistance cassette was removed from QKO plants. Protoplasts of QKO plants were transiently transformed by a Cre-expressing cassette (generous gift from Mitsuyasu Hasebe, National Institute for Basic Biology, Okazaki, Japan) by PEG-mediated transformation as described above. The protoplasts were grown on regenerating media without selection. A portion of regenerated filaments was transferred onto hygromycincontaining media to confirm the deletion of the hygromycin-resistance cassette. This QKO (-hyg) was used for PEG-mediated transformation by the constructs to obtain the complemented lines.

In-gel phosphorylation assay. One-week-old protonemata of wild-type or $P p S n R K 2$ disruptants were treated with $10 \mu \mathrm{M}$ ABA or $0.4 \mathrm{M}$ mannitol for indicated periods and subjected to in-gel phosphorylation assays. Tissues were powdered using a Tissuelyser (QIAGEN) in liquid nitrogen and homogenized in extraction buffer containing $50 \mathrm{mM} \beta$-glycerophosphate, $50 \mathrm{mM}$ HEPES pH 7.5, $5 \mathrm{mM}$ EDTA $2 \mathrm{mM}$ PMSF, $5 \mathrm{mM}$ EGTA, $2 \mathrm{mM}$ DTT, $1 \mathrm{mM} \mathrm{Na} 3 \mathrm{VO}_{4}, 25 \mathrm{mM} \mathrm{NaF}, 2 \mathrm{mg} \mathrm{ml}^{-1}$ leupeptin, $2 \mathrm{mg} \mathrm{ml}^{-1}$ pepstatin A, and $20 \%$ glycerol. Equal amounts of total soluble proteins were electrophoresed in $10 \%$ SDS-polyacrylamide gel containing $0.5 \%\left(\mathrm{w} \mathrm{v}^{-1}\right)$ histone IIIS as substrates. After denaturation and renaturation procedures, the kinase activity was detected by incubation with $50 \mu \mathrm{M} \gamma-{ }^{32} \mathrm{P}$-labeled 
ATP in a buffer containing $2 \mathrm{mM}$ DTT, $40 \mathrm{mM}$ Hepes- $\mathrm{KOH}$ (pH 7.5), $0.1 \mathrm{mM}$ EGTA and $5 \mathrm{mM} \mathrm{MgCl}_{2}$, followed by washing ${ }^{20}$ and visualization of the blots was done by autoradiography or BAStation 2500 (Fuji Film),

Tests of stress tolerance. The osmostress treatment was carried out by soaking the one-week-old protonemata in indicated concentrations of mannitol solution for $15 \mathrm{~min}$, then washed with water. The survival of protonemal cells was checked by Evans Blue staining or cultured on BCDAT medium without mannitol for one week. For the evaluation of dehydration tolerance, protonemata were treated with or without $\mathrm{ABA}$ for $24 \mathrm{~h}$, then transferred to an empty Petri dish and dehydrated inside a sealed chamber in atmospheres of known relative humidity (RH), generated by saturated salt solutions; these were placed in an incubator at $25^{\circ} \mathrm{C}$ with a continuous light for $24 \mathrm{~h}$. These tissues were then rehydrated by incubating under the standard growth condition described above for two weeks. Saturated salt solutions were used to generate constant $\mathrm{RH}$ at $25^{\circ} \mathrm{C}$ and the corresponding water potential values were $75.3 \% \mathrm{RH}$ and $-39 \mathrm{MPa}$ for $\mathrm{NaCl}$ and $95 \% \mathrm{RH}$ and $-4 \mathrm{MPa}$ for $\mathrm{K}_{2} \mathrm{SO}_{4}$, respectively ${ }^{17}$.

Protein analysis. Protonemata were incubated either with or without 1 and $10 \mu \mathrm{M}$ $\mathrm{ABA}$, or 0.25 and $0.5 \mathrm{M}$ mannitol for one day. Boiling-soluble fractions of the extracted proteins were separated by SDS-polyacrylamide gel and stained with Coomassie Brilliant Blue. Boiling-soluble protein fractions were subjected to immunoblot analysis using antibody against the LEA-like protein 17B9. The antibody-reacted proteins were visualized by reaction with the chemiluminescent reagent and exposure to X-ray film. The anti-17B9 (anti-rabbit antiserum against recombinant 17B9 protein purified from E. coli) and the anti-rabbit IgG HRP (\#458, MBL Life science, Japan) were used at 1:1000 and 1:10000 dilutions, respectively. All full-length blots and gels are shown in Supplementary Figures $16-23$

Transient assay. Each of the coding sequences for PpSnRK2 genes, for KnSnRK2 or for Arabidopsis $S n R K 2.5, \operatorname{SnRK2.6}$, and $S n R K 2.8$ were cloned into the expression cassette controlled by the PpSnRK2D native promoter ${ }^{51}$. These plasmids were subjected to the transient assay using particle bombardment of protonemata ${ }^{51}$. We used $0.8 \mu \mathrm{g}$ each of the reporter constructs (Em-GUS or PpLEA1-GUS and Ubi$L U C$ ) and the effector construct to prepare DNA-coated gold particles for four shots. One-week-old protonemal tissue was used and incubated on BCDAT agar medium with or without $10 \mu \mathrm{M} \mathrm{ABA}$ or $0.4 \mathrm{M}$ mannitol for $24 \mathrm{~h}$. GUS activity was normalized by the luciferase activity and represented as relative GUS activity (GUS per LUC).

Transcriptomic analysis. Protonemata were cultured for one week under normal conditions as described above. For osmostress and ABA treatments, the protonemata were transferred to fresh medium, supplemented with $0.4 \mathrm{M}$ mannitol, while for ABA treatments, ABA was added to a final concentration of $10 \mu \mathrm{M}$. After $12 \mathrm{~h}$, the protonemata were harvested. Total RNA was extracted from the protonemal tissues using the RNeasy plant mini Kit (QIAGEN). The RNA-seq libraries were prepared using the TruSeq RNA sample prep kit v2 (Illumina) in accordance with manufacturer's instructions. Library quality control was performed with a Bioanalyzer 2100 (Agilent). The libraries included inserts that ranged in size from $\sim 200-500$ bp. The average insert size in the library was approximately $300 \mathrm{bp}$. Library quantification was performed with quantitative real-time PCR, and then the concentration of each library was adjusted to $10 \mathrm{nM}$. The prepared libraries were sequenced using the Illumina HiSeq 2500 system (Illumina). The raw reads for each library were deposited in DDBJ and are accessible through Sequence Read Archive accession number DRA005879. Paired-end reads from each library were processed using CASAVA version 1.8.2 in FASTQ format. The FASTQ files were imported to the CLC Genomics Workbench (QIAGEN) for the following analysis. The trim sequences tool in the suite was used to remove adapters and filter out lowquality bases $(<\mathrm{Q} 13)$, and only those reads that showed a quality score of 13 or higher were retained. Filtered sequence reads were mapped onto the $P$. patens genome v3.3 (Phytozome 11; https://phytozome.jgi.doe.gov) using CLC assembly with default parameters. Quality control was carried out with filtering for the number of mapped reads in the exon region; genes with fewer than 5 mapped reads in any samples were removed. After filtering, a total of 16,924 genes were subjected to further analysis. Expression values were reported as RPKM values. Differentially expressed genes were detected by empirical analysis with the DGE algorithm in CLC software. The results were filtered based on a false discovery rate (FDR) with $\mathrm{P}$ values less than 0.05 and a corrected fold change greater than 2. Principal component analysis (PCA) was performed using the "Principal component analysis" function in CLC software, and the results are shown as a scatterplot with principal components as the axes. Hierarchical clustering and heatmap analysis were performed based on Euclidian distance in the CLC software. The RPKM values were used for both PCA and the hierarchical clustering analysis. Gene ontology enrichment analysis was performed using BiNGO (Biological Network Gene Ontology) software, a Cytoscape plug-in (available at www.cytoscape.org) for putative Arabidopsis orthologues of SAGs. These putative Arabidopsis orthologues were identified by a blastp search against the TAIR10 protein database. The degree of functional enrichment for a given cluster was quantitatively assessed by a hypergeometric test, and a multiple test correction was applied using the FDR algorithm implemented in the BiNGO plug-in. Overrepresented gene ontology terms were generated after FDR correction, with a significance level of 0.05 .

Phosphoproteomic analyses. $P$. patens wild type and SnRK2 disrupted mutants were treated with $10 \mu \mathrm{M}$ ABA for $0,15,30$ and 90 min. Plant samples were homogenized in protein extraction buffer containing $10 \mathrm{mM}$ Tris- $\mathrm{HCl}(\mathrm{pH} 9.0)$, $8 \mathrm{M}$ Urea, 2\% Phosphatase Inhibitor Cocktail II (Sigma) and 2\% Phosphatase Inhibitor Cocktail III (Sigma), and then centrifuged at 15,000 r.p.m for $10 \mathrm{~min}$ on $4{ }^{\circ} \mathrm{C}$ to obtain crude extracts. Aliquots of crude extract (500 $\mu \mathrm{g}$ of total protein) were subjected to tryptic digestion and phosphopeptide enrichment with HAM$M{ }^{34}$. Enriched phosphopeptide samples were analyzed with a LC-MS/MS system, TripleTOF 5600 (SCIEX). Peptides and proteins were identified using the Physcomitrella patens database (Phytozome v12.1) with Mascot (Matrix Science) $)^{34}$ Skyline v3.7 (Maccoss lab software) was used for quantification of phosphopeptides on the basis of LC-MS peak area. The motif analysis was conducted using the Motif-X algorithm ${ }^{52}$.

Primers. Primer sequences used in this study are described in Supplementary Data 8.

Statistical analysis. Four biological repeats except for Fig. $4 \mathrm{~b}$ (three biological replicates) were conducted for the transient assays. Statistical significance between two samples was analyzed by two-tailed Student's $t$ test. The experiments were repeated at least three times on different occasions to confirm the reproducibility, and the representative experiments were presented. Error bars indicate the standard error. ${ }^{*} P<0.05,{ }^{*} P<0.01,{ }^{* *} P<0.001$.

Southern blot analysis. The genomic DNA of the Ppsnrk2 plants was extracted using PhytoPure (GE Healthcare) with an additional RNase treatment. The probes were amplified using a PCR DIG Probe Synthesis Kit (Roche Applied Science) with primers described in Suppelemtary Data 8 . Two $\mu$ g of genomic DNAs were digested with restriction enzymes and were separated on $0.6 \%$ agarose gels and transferred to nylon filters (Hybond-N, GE Healthcare). The membrane was hybridized with DIG-labelled probes in DIG Easy Hyb buffer (Roche Applied Science) at $41^{\circ} \mathrm{C}$ overnight. After washing, the membrane was treated once with blocking buffer for $1 \mathrm{~h}$ at room temperature. The membrane was reacted with AntiDig (Roche Applied Science) for $1 \mathrm{~h}$ at room temperature. Detection was carried out with CDP-Star (Roche Applied Science) for $5 \mathrm{~min}$ at room temperature in the dark. The signal was detected by ChemiDoc XRS Plus (BIO-RAD).

Quantitative RT-PCR. First-strand complementary DNA was synthesized from 0.5 ug total RNA subjected to RNA-seq analysis using a ReverTra Ace qPCR RT Master Mix with gDNA Remover (TOYOBO). qRT-PCR was performed using LightCycler 96 (Roche Diagnostics). THUNDERBIRD SYBR qPCR Mix (TOYOBO) was used for amplification. Ubiquitin-conjugating enzyme E2 (Pp3c14_21480) was used as an internal standard. Primers are listed in Supplementary Data 8.

In vitro phosphorylation assay. For in vitro phosphorylation assay of PpSnRK2D by ARK, cDNA of PpSnRK2D with or without mutations was subcloned into the pMAL-c5X vector (New England Biolabs) and expressed in Escherichia coli (BL21) The MBP-fused PpSnRK2 proteins were purified by amylose resin. The vector expressing ARK-KD ${ }^{23}$ was expressed in Escherichia coli (BL21). GST-fused ARKKD protein was purified by glutathione-Sepharose resin. Both recombinant proteins were subjected to ultrafiltration using a Nanosep 30-kDa size-exclusion column (Pall) for concentration and removal of low-molecular-weight materials. In vitro phosphorylation reactions were carried out using the recombinant proteins by incubating at $30^{\circ} \mathrm{C}$ for $30 \mathrm{~min}$ in the reaction buffer ${ }^{23}$ in the presence of $\gamma-32 \mathrm{P}$ ATP. The reaction mixture was separated by SDS-PAGE and phosphorylated protein detected by autoradiography. For in vitro phosphorylation assay of $\mathrm{ABA}$ and PpSnRK2-regulated phosphopeptides by PpSnRK2B, we selected 3 peptides, of which phosphorylation levels were decreased in ABA-treated QKO in phosphoproteome analysis and designed primers to synthesise 33 amino acid sequences in total for each peptide with a phosphorylation site near the center, and GST-fused proteins were prepared using pGEX-5X-3 vector in Escherichia coli (KRX). The GST-fusion peptides were purified as described above, and GST protein was used as a negative control.

Immunoblot assay. Total proteins from $P$. patens wild-type and Ppsnrk2 plants were extracted with buffer (50 mM HEPES pH 7.5, 5 mM EDTA, 5 mM EGTA, $25 \mathrm{mM} \mathrm{NaF}, 2 \mathrm{mM}$ DTT, $1 \mathrm{mM} \mathrm{Na}_{3} \mathrm{VO}_{4}, 2 \mathrm{mg} \mathrm{ml}^{-1}$ leupeptin, $2 \mathrm{mg} \mathrm{ml}^{-1}$ pepstatin A, 2 mM PMSF, $50 \mathrm{mM}$ b-glycerophosphate, and $20 \%$ glycerol). Equal amounts of total soluble proteins were electrophoresed in 10\% SDS-polyacrylamide gel. The proteins were detected by the anti-HA-tag pAb (\#561, MBL Life science). Detection of the antibody-reacted proteins were carried out with Amersham ECL Prime Western Blotting Detection Reagent (GE Healthcare). The signal was detected by ChemiDoc XRS Plus (BIO-RAD). The anti-HA-tag pAb and the anti- 
rabbit IgG $(\mathrm{H}+\mathrm{L})(\# 170-6515$, BIO-RAD) were used at 1:2000 and 1:50000 dilutions, respectively.

RNA blot analysis. Total RNAs were extracted from protonemal tissues of wildtype and ppsnrk2 plants treated with or without $10 \mu \mathrm{M}$ ABA for indicated periods using the RNeasy plant mini Kit (QIAGEN). The primer sets of probes are described in Supplementary Data 8. Hybridization was carried out with ${ }^{32} \mathrm{P}$-labelled DNA probes. The signal was detected by BAStation 2500 (Fuji Film).

Measurement of freezing tolerance. One-week old protonemata of wild-type and Ppsnrk2 plants were treated with or without $10 \mu \mathrm{M} \mathrm{ABA}$ for $24 \mathrm{~h}$ and subjected to freezing at $-5^{\circ} \mathrm{C}$. Freezing tolerance was determined by measurement of electrolyte leakage. The amount of electrolyte leakage from the freeze-thawed tissues (Eft) and the amount of electrolytes after boiling $(\mathrm{Eb})$ were measured. Survivals of the cells were represented as the Eft per Eb ratios (\%).

\section{Data availability}

Physcomitrella Sequence data of SnRK2 genes and other genes used in this study can be found from Phytozome (DOE-JGI, http://phytozome.jgi.doe.gov/). The RNA-seq data are deposited to DDBJ Sequence Read Archive (DRA) with the accession number DRA005879. All raw data files for phosphoproteome were deposited in the Japan Proteome Standard Repository/Database (jPOST; PST000493). Data source of amino acid sequences of SnRK2-type protein kinases used for phylogenetic analysis is available in Supplementary Data 9. Source data for all figures presented in the manuscript are available in Supplementary Data 10. All other data supporting the results of this study are available from the authors upon request.

Received: 26 March 2018 Accepted: 28 December 2018

Published online: 21 January 2019

\section{References}

1. Rock, C. D., Sakata, Y. \& Quatrano, R. S. in Abiotic Stress Adaptation in Plants (eds Pareek, A. et al.) 33-73 (Springer, Netherlands, 2009).

2. Umezawa, $\mathrm{T}$. et al. Type $2 \mathrm{C}$ protein phosphatases directly regulate abscisic acid-activated protein kinases in Arabidopsis. Proc. Natl Acad. Sci. USA 106, 17588-17593 (2009).

3. Fujii, H. \& Zhu, J.-K. Arabidopsis mutant deficient in 3 abscisic acid-activated protein kinases reveals critical roles in growth, reproduction, and stress. Proc. Natl Acad. Sci. USA 106, 8380-8385 (2009).

4. Nakashima, K. et al. Three Arabidopsis SnRK2 protein kinases, SRK2D/ SnRK2.2, SRK2E/SnRK2.6/OST1 and SRK2I/SnRK2.3, involved in ABA signaling are essential for the control of seed development and dormancy. Plant Cell Physiol. 50, 1345-1363 (2009).

5. Fujita, Y. et al. Three SnRK2 protein kinases are the main positive regulators of abscisic acid signaling in response to water stress in Arabidopsis. Plant Cell Physiol. 50, 2123-2132 (2009).

6. Umezawa, T. et al. Molecular basis of the core regulatory network in ABA responses: sensing, signaling and transport. Plant Cell Physiol. 51, 1821-1839 (2010).

7. Park, S.-Y. et al. Abscisic acid inhibits type $2 \mathrm{C}$ protein phosphatases via the PYR/PYL family of START proteins. Science 324, 1068-1071 (2009).

8. $\mathrm{Ma}, \mathrm{Y}$. et al. Regulators of PP2C phosphatase activity function as abscisic acid sensors. Science 324, 1064-1068 (2009).

9. Vlad, F. et al. Protein phosphatases $2 \mathrm{C}$ regulate the activation of the Snf1related kinase OST1 by abscisic acid in Arabidopsis. Plant Cell 21, 3170-3184 (2009).

10. Fujii, H. et al. In vitro reconstitution of an abscisic acid signalling pathway. Nature 462, 660-664 (2009).

11. Fujii, H., Verslues, P. E. \& Zhu, J.-K. Arabidopsis decuple mutant reveals the importance of SnRK2 kinases in osmotic stress responses in vivo. Proc. Natl Acad. Sci. USA 108, 1717-1722 (2011).

12. Takezawa, D., Komatsu, K. \& Sakata, Y. ABA in bryophytes: how a universal growth regulator in life became a plant hormone? J. Plant. Res. 124, 437-453 (2011).

13. Rensing, S. A. et al. The Physcomitrella genome reveals evolutionary insights into the conquest of land by plants. Science 319, 64-69 (2008).

14. Chater, C. et al. Regulatory mechanism controlling stomatal behavior conserved across 400 million years of land plant evolution. Curr. Biol. 21, 1025-1029 (2011).

15. Knight, C. D. et al. Molecular responses to abscisic acid and stress are conserved between moss and cereals. Plant Cell 7, 499-506 (1995).
16. Kamisugi, Y. \& Cuming, A. C. The evolution of the abscisic acid-response in land plants: comparative analysis of group 1 LEA gene expression in moss and cereals. Plant Mol. Biol. 59, 723-737 (2005).

17. Koster, K. L., Balsamo, R. A., Espinoza, C. \& Oliver, M. J. Desiccation sensitivity and tolerance in the moss Physcomitrella patens: assessing limits and damage. Plant Growth Regul. 62, 293-302 (2010).

18. Khandelwal, A. et al. Role of ABA and ABI3 in desiccation tolerance. Science 327, 546-546 (2010).

19. Minami, A. et al. Cold acclimation in bryophytes: low-temperature-induced freezing tolerance in Physcomitrella patens is associated with increases in expression levels of stress-related genes but not with increase in level of endogenous abscisic acid. Planta 220, 414-423 (2005).

20. Komatsu, K. et al. Group A PP2Cs evolved in land plants as key regulators of intrinsic desiccation tolerance. Nat. Commun. 4, 2219 (2013).

21. Stevenson, S. R. et al. Genetic analysis of Physcomitrella patens identifies ABSCISIC ACID NON-RESPONSIVE (ANR), a regulator of ABA responses unique to basal land plants and required for desiccation tolerance. Plant Cell 28, 1310-1327 (2016).

22. Yasumura, Y. et al. An ancestral role for constitutive triple response 1 (CTR1) proteins in both ethylene and abscisic acid signaling. Plant Physiol. 169, 283-298 (2015).

23. Saruhashi, M. et al. Plant Raf-like kinase integrates abscisic acid and hyperosmotic stress signaling upstream of SNF1-related proteinkinase2. Proc. Natl Acad. Sci. USA 112, 201511238-E6396 (2015).

24. Hori, K. et al. Klebsormidium flaccidum genome reveals primary factors for plant terrestrial adaptation. Nat. Commun. 5, 3978 (2014).

25. Goode, J. A., Stead, A. D. \& Duckett, J. G. Redifferentiation of moss protonemata: an experimental and immunofluorescence study of brood cell formation. Can. J. Bot. 71, 1510-1519 (1993).

26. Schnepf, E. \& Reinhard, C. Brachycytes in Funaria protonemata: induction by abscisic acid and fine structure. J. Plant. Physiol. 151, 166-175 (1997).

27. Minami, A., Nagao, M., Arakawa, K., Fujikawa, S. \& Takezawa, D. Abscisic acid-induced freezing tolerance in the moss Physcomitrella patens is accompanied by increased expression of stress-related genes. J. Plant. Physiol. 160, 475-483 (2003).

28. Yotsui, I. et al. Large-scale proteome analysis of abscisic acid and ABSCISIC ACID INSENSITIVE3-dependent proteins related to desiccation tolerance in Physcomitrella patens. Biochem. Biophys. Res. Commun. 471, 589-595 (2016).

29. Shih, M. D., Hoekstra, F. A. \& Hsing, Y. I. C. Chapter 4 late embryogenesis abundant proteins. Adv. Bot. Res. 48, 211-255 (2008).

30. Takezawa, D. et al. Epoxycarotenoid-mediated synthesis of abscisic acid in Physcomitrella patens implicating conserved mechanisms for acclimation to hyperosmosis in embryophytes. New Phytol. 206, 209-219 (2015).

31. Lavy, M. et al. Constitutive auxin response in Physcomitrella reveals complex interactions between Aux/IAA and ARF proteins. eLife 5, 427 (2016).

32. Chang, C.-Y., Lin, W.-D. \& Tu, S.-L. Genome-wide analysis of heat-sensitive alternative splicing in Physcomitrella patens. Plant Physiol. 165, 826-840 (2014).

33. Frank, M. H. \& Scanlon, M. J. Transcriptomic evidence for the evolution of shoot meristem function in sporophyte-dominant land plants through concerted selection of ancestral gametophytic and sporophytic genetic programs. Mol. Biol. Evol. 32, 3033-3033 (2015).

34. Umezawa, T. et al. Genetics and phosphoproteomics reveal a protein phosphorylation network in the abscisic acid signaling pathway in Arabidopsis thaliana. Sci. Signal. 6, rs8-rs8 (2013).

35. Amagai, A. et al. Phosphoproteomic profiling reveals ABA-responsive phosphosignaling pathways in Physcomitrella patens. Plant J. 94, 699-708 (2018).

36. Kobayashi, Y. et al. Abscisic acid-activated SNRK2 protein kinases function in the gene-regulation pathway of ABA signal transduction by phosphorylating ABA response element-binding factors. Plant J. 44, 939-949 (2005).

37. Furihata, T. et al. Abscisic acid-dependent multisite phosphorylation regulates the activity of a transcription activator AREB1. Proc. Natl Acad. Sci. USA 103, 1988-1993 (2006).

38. Vlad, F., Turk, B. E., Peynot, P., Leung, J. \& Merlot, S. A versatile strategy to define the phosphorylation preferences of plant protein kinases and screen for putative substrates. Plant J. 55, 104-117 (2008).

39. Wang, P. et al. Quantitative phosphoproteomics identifies SnRK2 protein kinase substrates and reveals the effectors of abscisic acid action. Proc. Natl Acad. Sci. USA 110, 11205-11210 (2013).

40. Marella, H. H., Sakata, Y. \& Quatrano, R. S. Characterization and functional analysis of ABSCISIC ACID INSENSITIVE3-like genes from Physcomitrella patens. Plant J. 46, 1032-1044 (2006).

41. Yotsui, I. et al. ABSCISIC ACID INSENSITIVE3 regulates abscisic acidresponsive gene expression with the nuclear factor Y complex through the ACTT-core element in Physcomitrella patens. New Phytol. 199, 101-109 (2013). 
42. Boudsocq, M., Droillard, M.-J., Barbier-Brygoo, H. \& Laurière, C. Different phosphorylation mechanisms are involved in the activation of sucrose nonfermenting 1 related protein kinases 2 by osmotic stresses and abscisic acid. Plant Mol. Biol. 63, 491-503 (2007).

43. Hauser, F., Waadt, R. \& Schroeder, J. I. Evolution of abscisic acid synthesis and signaling mechanisms. Curr. Biol. 21, R346-R355 (2011).

44. Kenrick, P. \& Crane, P. R. The origin and early evolution of plants on land. Nature 389, 33-39 (1997).

45. Karol, K. G., McCourt, R. M., Cimino, M. T. \& Delwiche, C. F. The closest living relatives of land plants. Science 294, 2351-2353 (2001).

46. Wickett, N. J. et al. Phylotranscriptomic analysis of the origin and early diversification of land plants. Proc. Natl Acad. Sci. USA 111, E4859-E4868 (2014).

47. Becker, B. \& Marin, B. Streptophyte algae and the origin of embryophytes. Ann. Bot. 103, 999-1004 (2009).

48. Holzinger, A. \& Pichrtová, M. Abiotic stress tolerance of charophyte green algae: new challenges for omics techniques. Front. Plant Sci. 7, 678 (2016)

49. Harholt, J., Moestrup, Ø. \& Ulvskov, P. Why plants were terrestrial from the beginning. Trends Plant. Sci. 21, 96-101 (2016).

50. Hartung, W. The evolution of abscisic acid (ABA) and ABA function in lower plants, fungi and lichen. Funct. Plant Biol. 37, 806-812 (2010).

51. Komatsu, K. et al. Functional analyses of the ABI1-related protein phosphatase type $2 \mathrm{C}$ reveal evolutionarily conserved regulation of abscisic acid signaling between Arabidopsis and the moss Physcomitrella patens. Plant Mol. Biol. 70, 327-340 (2009).

52. Chou, M. F. \& Schwartz, D. Biological sequence motif discovery using motif-x. Curr. Protoc. Bioinforma. Chapter 13, Unit13.15-24 (2011).

\section{Acknowledgements}

We thank Ralph Quatrano for Em-GUS, Tuan-hua David Ho for Ubi-LUC, Marika Arai and Saho Mizukado for technical assistance, and the NODAI GenomeResearch Center of Tokyo University of Agriculture for the use of Illumina HiSeq 2500. This study was supported in part by Grants-in-Aid for Scientific Research (No. 26291054 and No. 16H01460 to D.T., and No. 15547992 to T.U.) from the Ministry of Education, Culture, Sports, Science, and Technology of Japan (MEXT); and by Precursory Research for Embryonic Science and Technology (PRESTO) from Japan Science and Technology Agency (T.U.).

\section{Author contributions}

A.S., R.O. D.T. Y.K. and A.C.C. generated the transgenic plants and performed physiological experiments. K.K. and K.T. performed RNA-seq analysis. A.A, S.I., Y.H., F.T., K.S. and T.U. performed phosphoproteome analysis. K.H. and H.O. prepared cloned SnRK2 gene from K. nitens. T.T., T.H., and Y.S. designed the experiments and wrote the manuscript.

\section{Additional information}

Supplementary information accompanies this paper at https://doi.org/10.1038/s42003 019-0281-1.

Competing interests: The authors declare no competing interests.

Reprints and permission information is available online at http://npg.nature.com/ reprintsandpermissions/

Publisher's note: Springer Nature remains neutral with regard to jurisdictional claims in published maps and institutional affiliations.

cc (i) Open Access This article is licensed under a Creative Commons Attribution 4.0 International License, which permits use, sharing, adaptation, distribution and reproduction in any medium or format, as long as you give appropriate credit to the original author(s) and the source, provide a link to the Creative Commons license, and indicate if changes were made. The images or other third party material in this article are included in the article's Creative Commons license, unless indicated otherwise in a credit line to the material. If material is not included in the article's Creative Commons license and your intended use is not permitted by statutory regulation or exceeds the permitted use, you will need to obtain permission directly from the copyright holder. To view a copy of this license, visit http://creativecommons.org/ licenses/by/4.0/.

(C) The Author(s) 2019 